\title{
On the influence of the thickness of the sediment moving layer in the definition of the bedload transport formula in Exner systems
}

\author{
E.D. Fernández-Nieto ${ }^{\mathrm{a}}$, C. Lucas ${ }^{\mathrm{b}}$, T. Morales de Luna ${ }^{\mathrm{c}, *}$, S. Cordier ${ }^{\mathrm{b}}$ \\ ${ }^{a}$ Dpto. Matemática Aplicada I. ETS Arquitectura - Universidad de Sevilla. \\ Avda. Reina Mercedes N. 2. 41012-Sevilla, Spain \\ ${ }^{b}$ MAPMO UMR CNRS 7349, Université d'Orléans, UFR Sciences, Bâtiment de mathématiques, \\ B.P. 6759 - 45067 Orléans cedex 2, France \\ ${ }^{c}$ Dpto. de Matemáticas. Universidad de Córdoba. Campus de Rabanales. 14071 Córdoba, Spain
}

\begin{abstract}
In this paper we study Exner system and introduce a modified general definition for bedload transport flux. The new formulation has the advantage of taking into account the thickness of the sediment layer which avoids mass conservation problems in certain situations. Moreover, it reduces to a classical solid transport discharge formula in the case of quasi-uniform regime. We also present several numerical tests where we compare the proposed sediment transport formula with the classical formulation and we show the behaviour of the new model in different configurations.
\end{abstract}

\section{Introduction}

We are interested in the study of sediment transport in shallow water regimes. As it is said by Simons et al. in [1], sediment particles are transported by flow in one or a combination of ways: rolling or sliding on the bed (surface creep), jumping into the flow and then resting on the bed (saltation), and supported by the surrounding fluid during a significant part of its motion (suspension) (See Figure 1). There is no sharp line between saltation and suspension, and sediments may be transported partially by saltation and then suddenly be caught by the flow turbulence and transported in suspension. However, this distinction is important as it serves to delimit two methods of hydraulic transportation which follow different laws, i.e., traction and suspension. This means that sediment transport occurs in two main modes: bedload and suspended load. Here we are going to focus on bedload transport and neglect suspension. The bedload is the part of the total load which is travelling immediately above the bed and is supported by intergranular collisions rather than fluid turbulence (see [2]). The suspended load, on the other hand, is the part of the load which is primarily supported by the fluid turbulence (c.f. [3]). Thus, bedload includes mainly sediment transport for coarse materials (saltation) or fine material on plane beds (saltation at low shear stresses and sheet flow at high shear stresses), although both types of transport can occur together and the limit is not always easy to define.

\footnotetext{
${ }^{*}$ Corresponding author

Email addresses: edofer@us.es (E.D. Fernández-Nieto), carine.lucas@univ-orleans.fr (C. Lucas), tomas.morales@uco.es (T. Morales de Luna), stephane.cordier@math.cnrs.fr (S. Cordier)
} 


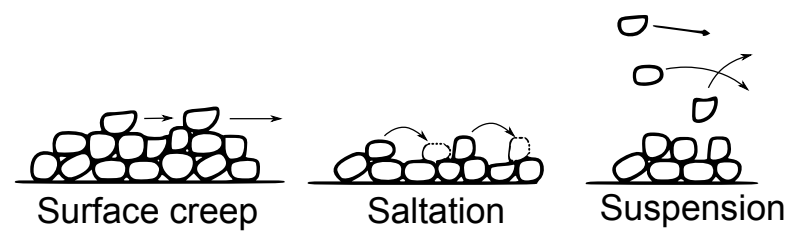

Figure 1: Transport of particles by a flow

In this context of bedload, we are interested in writing an erosion-deposition model (see for example [4], [5]). One could assume that the sediment layer can be decomposed in two layers: a layer that moves due to the action of the river, whose thickness is denoted by $z_{m}$, and a layer composed by sediments that are not moving but are susceptible to move and denoted by $z_{f}$. In other words, particles within the layer $z_{f}$ can be eroded and transferred to the moving layer $z_{m}$ and particles moving within the layer $z_{m}$ can stop and be deposed on the layer $z_{f}$. In general, if $z_{b}$ is the thickness of the erodible bed we have the relation (see Figure 2),

$$
z_{b}=z_{m}+z_{f}
$$

The sediment layer is supposed to stay on a non-erodible fixed layer of thickness $z_{r}$ which is usually called the bedrock layer.

Let us denote by $\dot{z}_{e}$ the erosion rate and by $\dot{z}_{d}$ the deposition rate (see Subsection 2.2 for details). In this situation, we may consider the following coupled system of PDE,

$$
\left\{\begin{array}{l}
\partial_{t} h+\partial_{x}(h u)=0, \\
\partial_{t}(h u)+\partial_{x}\left(h u^{2}+g h^{2} / 2\right)=-g h \partial_{x}\left(z_{m}+z_{f}+z_{r}\right), \\
\partial_{t} z_{m}+\partial_{x} \widehat{q}_{b}=\dot{z}_{e}-\dot{z}_{d}, \\
\partial_{t} z_{f}=-\dot{z}_{e}+\dot{z}_{d},
\end{array}\right.
$$

where $h$ is the water depth, $u$ is the flow velocity. $\widehat{q_{b}}$ is the volumetric bedload sediment transport rate per unit time and width, described by some empirical law. The sediment layer is usually assumed to be a porous layer with porosity $\varphi$ and the definition of $\widehat{q_{b}}$ includes the division by $(1-\varphi)$. Finally, $g$ is the acceleration due to gravity. The conservative variable $h u$ is also called water discharge and noted by $q$.

Note that in some situations the time scale associated with the bed deformation is very long compared with the time scale of the variations of the fluid. In these cases two time scales systems could be considered, as in [6]. This is not the approach we chose in this paper because it may not be true in general. In fact, many time scales can be identified in the sediment transport process (see for example [7]). 


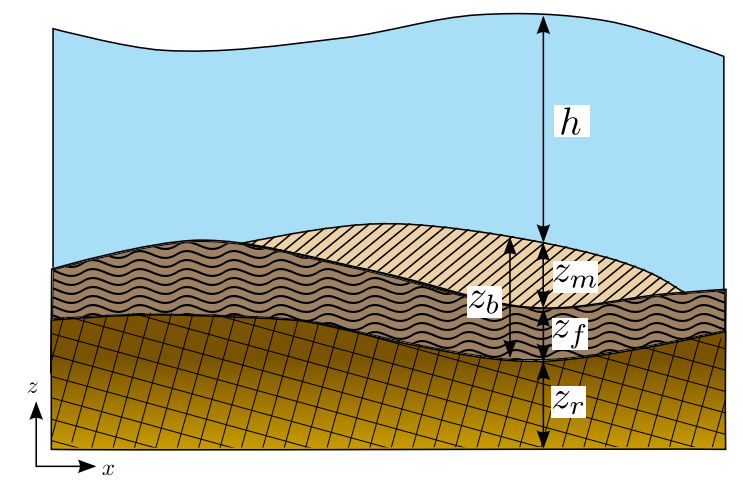

Figure 2: Sketch of shallow water over an erodible bed

Let us remark that, by adding up the last two equations in (2) we recover a mass conservation law also called Exner equation [8] in the context of bedload transport (see for example [9, 10, 11, 12]). Then, the Shallow Water-Exner system (2) may be written in the form

$$
\left\{\begin{array}{l}
\partial_{t} h+\partial_{x}(h u)=0 \\
\partial_{t}(h u)+\partial_{x}\left(h u^{2}+g h^{2} / 2\right)=-g h \partial_{x}\left(z_{b}+z_{r}\right), \\
\partial_{t} z_{b}+\partial_{x} \widehat{q_{b}}=0 .
\end{array}\right.
$$

Despite the strong simplification hypotheses used in the derivation of the model and the lack of some good mathematical properties of the PDE system obtained (there is neither a momentum equation for the sediment layer nor an entropy pair) the approach of the bedload transport by means of an empirical solid transport discharge formula is widely spread for practical purposes: see for example [13], [14], [15], [8], [16].

In general, the solid transport discharge may depend on all the unknowns $\widehat{q_{b}}=\widehat{q_{b}}\left(h, h u, z_{b}, z_{f}\right)$ but classical formulae for this bedload transport only depend on the hydrodynamical variables $h$ and $u$ (see Subsection 2.1). In these cases, instead of solving system (2), it is enough to consider the reduced system (3). With the purpose to distinguish whether solid transport discharge depends only on the hydrodynamical variables or on all the variables, we use the following notation:

$$
\widehat{q_{b}}=\left\{\begin{array}{l}
q_{b} \equiv q_{b}(h, h u), \\
\widetilde{q_{b}} \equiv \widetilde{q_{b}}\left(h, h u, z_{b}, z_{f}\right) .
\end{array}\right.
$$

But, let us remark that if we consider a classical formula for solid transport, $\widehat{q_{b}}=q_{b}(h, h u)$, the mass conservation law for the sediment layer may fail. For instance, let us consider a situation like the one described in Figure 3, where the sediment layer is only present in the interior of the domain.

Suppose that no sediment comes in or out of the domain through the boundaries during the time interval $[0, T]$. By integrating the third equation of $(3)$ over $[0, T] \times[a, b]$, we obtain 


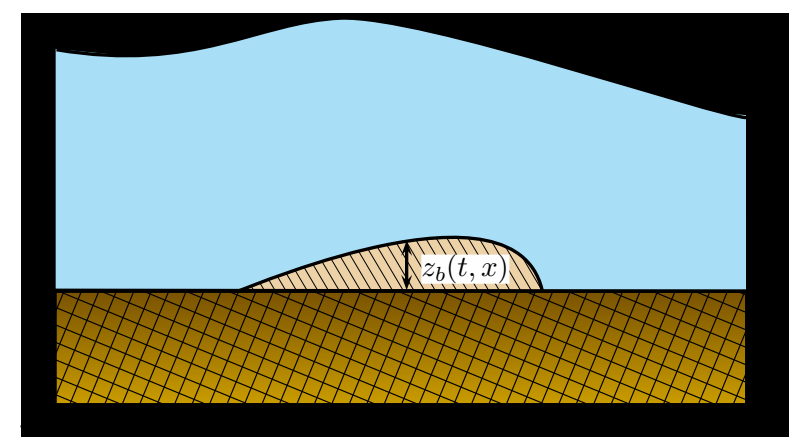

Figure 3: Sediment layer isolated in the interior of the domain

$$
\left.\int_{a}^{b}\left(z_{b}\right)\right|_{t=T} d x-\left.\int_{a}^{b}\left(z_{b}\right)\right|_{t=0} d x=-\int_{0}^{T}\left(\left.\widehat{q_{b}}\right|_{x=b}-\left.\widehat{q_{b}}\right|_{x=a}\right) d t .
$$

Let us suppose that the solid flux $\widehat{q_{b}}$ does not take into account the sediment layer thickness and only depends on the variables $h$ and $h u$, i.e. $\widehat{q_{b}}=q_{b}$. Assume a velocity high enough in order to have that $q_{b}$ is non-zero, and that $h$ and $h u$ are not equal at the boundaries $x=a$ and $x=b$. Then we will have $\left.q_{b}\right|_{x=a} \neq\left. q_{b}\right|_{x=b}$ and non-zero. Thus, in a situation like the one described in Figure 3 the right hand side in (4) may be non zero. This means that eventually the initial sediment mass is modified by an artificial flux at the boundary so that mass is not preserved.

Following the structure of a continuity equation, the solid discharge can be written in the form

$$
\widehat{q_{b}}=z_{m} v_{b}, \quad\left(\text { recall that } z_{m}=z_{b}-z_{f},\right. \text { see equation (1)), }
$$

where $v_{b}$ is the bedload velocity. The problem is to find how to close the system by defining $v_{b}$. In fact, classical formulae define directly $\widehat{q_{b}}$ and not $v_{b}$ (see equations (7)-(12)) and the dependence on $z_{m}$ is not obvious due to several assumptions and simplifications.

In [17] Fowler et al. (see also [18]) propose a form to define $v_{b}$ in terms of a given classical transport discharge. Concretely, Fowler et al. propose a definition of $v_{b}$ in terms of the solid transport discharge proposed by Meyer-Peter \& Müller. This definition can be easily extended to any other classical bedload discharge as follows: suppose that $q_{b}(h, h u)$ is given by some classical empirical formula. Then, define

$$
\widehat{q_{b}}\left(h, h u, z_{b}, z_{f}\right)=\widetilde{q}_{b}\left(h, h u, z_{m}\right)=z_{m} v_{b} \quad \text { with } \quad v_{b}=\frac{q_{b}(h, h u)}{\bar{z}},
$$

where, $\bar{z}$ is a parameter of the model related to the mean value of the thickness of the sediment layer. In this paper we focus on this problem: the definition of the solid transport discharge $\widehat{q_{b}}$ in terms of the sediment thickness for a given classical bedload discharge formula. This paper is organized as follows: first, in Subsection 2.1, we review some of the classical deterministic transport discharge formulae and we consider a unified formulation. In Subsection 2.2 we present the definition of erosion 
and deposition rate terms. In Section 3 we propose a general formulation of the solid transport discharge. The proposed formula depends on the moving sediment layer, then the corresponding continuity equation preserves the sediment mass. Moreover, it reduces to a given classical solid transport discharge formula in the case of quasi-uniform regime. In Section 4 we study the influence of the variable $z_{b}$ on the eigenvalues of Exner system. First we show that Exner system is hyperbolic at least for physical situations. Second, we study the influence on the characterization of the sign of the eigenvalues of Exner system in terms of the hydraulic regime. In Section 5 we describe briefly the numerical approach for the simulation of the model by finite volume methods and we present some numerical results. Concretely, in Subsection 5.2 we compare the new sediment transport model introduced here with the classical formulation and we show the behaviour of the new model in different configurations (academic, realistic and also in comparison with experimental data).

\section{On the definition of classical bedload transport formulae, erosion and deposition rates}

\subsection{On the definition of classical bedload transport formulae}

Many different expressions of the solid transport discharge have been proposed in the literature. In this subsection we review some of the classical deterministic formulae and we consider a unified formulation (equation (13)).

The bedload transport formula proposed by Grass in [13] is among the simplest ones:

$$
q_{b}(h, h u)=\frac{A}{1-\varphi}\left|\frac{h u}{h}\right|^{m-1} \frac{h u}{h}=\frac{A}{1-\varphi}|u|^{m-1} u,
$$

where $A$ is the constant of interaction between the fluid and the sediment layer and $m$ is a parameter which is usually set to $m=3$.

In practice, estimations of bedload transport rate are mainly based on the bottom shear stress $\tau_{b}$, i.e. the force of water acting on the bed during its routing. The bottom shear stress is given as

$$
\tau_{b}=\rho g h S_{\mathrm{f}}
$$

where $\rho$ is the water density and $S_{\mathrm{f}}$ is the friction term that can be quantified by different empirical laws such as the Darcy-Weisbach or Manning formulae

- Darcy-Weisbach:

$$
S_{\mathrm{f}}=\frac{f u|u|}{8 g h},
$$

where $f$ is the Darcy-Weisbach coefficient.

- Manning:

$$
S_{\mathrm{f}}=\frac{n^{2} u|u|}{h^{4 / 3}},
$$

where $n$ is the Manning coefficient. 
The bottom shear stress is usually used in dimensionless form, noted $\tau_{b}^{*}$, which is also called Shields parameter. It is defined as the ratio between drag forces and the submerged weight by

$$
\tau_{b}^{*}=\frac{\left|\tau_{b}\right|}{\left(\rho_{\mathrm{s}}-\rho\right) g d_{\mathrm{s}}},
$$

where $\rho_{\mathrm{s}}$ is the sediment density and $d_{\mathrm{S}}$ is the diameter of sediment. The main hypothesis is that $\tau_{b}^{*}$ must exceed a threshold value $\tau_{\mathrm{cr}}^{*}$ in order to initiate motion. The threshold value $\tau_{\mathrm{cr}}^{*}$ depends on the physical properties of the sediment and is usually computed experimentally. One of the first works on this topic was done by Shields [19] in which $\tau_{\mathrm{cr}}^{*}$ is determined in relation with the boundary Reynolds number.

The bedload transport rate may be represented as a function of $\tau_{b}^{*}$ via a non-dimensional function $q_{b}^{*}$ by

$$
q_{b}=q_{b}^{*}\left(\tau_{b}^{*}\right) \frac{\operatorname{sgn}\left(\tau_{b}\right)}{1-\varphi} Q
$$

In the framework of the coupling with Shallow Water equation, the definition of $Q$ is chosen as turbulent. Then,

$$
Q=\sqrt{\left(\frac{\rho_{\mathrm{s}}}{\rho}-1\right) g d_{\mathrm{s}}^{3}} .
$$

The following expressions have been often applied [14, 20, 16]:

$$
\begin{aligned}
& \text { Meyer-Peter \& Müller (1948): } q_{b}^{*}\left(\tau_{b}^{*}\right)=8\left(\tau_{b}^{*}-\tau_{c r}^{*}\right)_{+}^{3 / 2}, \\
& \text { Fernández Luque \& Van Beek }(1976): q_{b}^{*}\left(\tau_{b}^{*}\right)=5.7\left(\tau_{b}^{*}-\tau_{c r}^{*}\right)_{+}^{3 / 2}, \\
& \text { Nielsen }(1992): q_{b}^{*}\left(\tau_{b}^{*}\right)=12 \sqrt{\tau_{b}^{*}}\left(\tau_{b}^{*}-\tau_{c r}^{*}\right)_{+}, \\
& \text {Ribberink (1998): } q_{b}^{*}\left(\tau_{b}^{*}\right)=11\left(\tau_{b}^{*}-\tau_{c r}^{*}\right)_{+}^{1.65}
\end{aligned}
$$

For turbulent flows, a characteristic value of $\tau_{c r}^{*}$ is 0.047 .

Note that these classical bedload transport formulae can be written under the unified form

$$
q_{b}(h, h u)=\frac{c}{1-\varphi}\left(\tau_{b}^{*}\right)^{m_{1}}\left(\tau_{b}^{*}-\tau_{c r}^{*}\right)_{+}^{m_{2}} \operatorname{sgn}\left(\tau_{b}\right) Q,
$$

where $c>0, m_{1} \geq 0$ and $m_{2} \geq 1$ are given constants, and $Q$ is defined by (8) for turbulent flows.

\subsection{On the definition of erosion and deposition rates}

In this subsection we specify the terms of the right hand side of system (2), that is, the erosion and deposition rate terms.

\section{Deposition rate}

In the literature we can find different definitions of the deposition rate $\dot{z}_{d}$, see for example [5], [21], [22]. We can write them under the uniform structure

$$
\dot{z}_{d}=K_{d} V \frac{z_{m}}{d_{s}},
$$


where $K_{d}$ is the deposition constant, $V$ is the characteristic velocity, that we can define as

$$
V=\frac{Q}{d_{s}}
$$

being $Q$ the characteristic discharge. Q is defined by (8).

\section{Erosion rate.}

If erosion is possible (i.e. $z_{f}>0$ ), the erosion rate can be related to the shear stress, through the relation:

$$
\dot{z}_{e}=K_{e} \frac{V}{(1-\varphi)}\left(\tau_{b}^{*}-\tau_{c r}^{*}\right)_{+},
$$

where $K_{e}$ is the erosion constant and $V$ is the characteristic velocity defined by (15).

In the following, we assume that $z_{f}$ does not vanish.

Remark 2.1. In [17], the erosion and deposition rates are defined in a different way, in terms of $\bar{z}, \bar{z}$ being a parameter of the model related to the mean value of the thickness of the sediment layer. It can be written under the following structure,

$$
\dot{z}_{e}-\dot{z}_{d}=K \frac{\bar{V}}{(1-\varphi)}\left(\tau_{b}^{*}-\tau_{c r}^{*}\right)_{+}^{3 / 2}\left(1-\frac{z_{m}}{\bar{z}}\right)_{+}, \quad \text { with } \quad \bar{V}=\sqrt{g d_{s}} \frac{d_{s}}{\bar{z}} \frac{\sqrt{\rho_{s}-\rho}}{\sqrt{\rho}}
$$

being $K$ a constant parameter.

\section{General formulation for the solid transport discharge}

The solid transport discharge formula for $\widehat{q_{b}}$ must be in agreement with the physics of the problem: if $z_{m}=0$, the solid transport discharge has to be 0 . In this section we propose a new general formulation of the solid transport discharge that takes into account the thickness of the sediment moving layer.

\subsection{Formulation}

Let us consider a classical bedload solid transport discharge, written in the form (13). Then, using the notation of the previous section, we define the following solid transport discharge,

$$
\widetilde{q}_{b}\left(h, h u, z_{m}\right)=z_{m} \alpha c\left(\tau_{b}^{*}\right)^{m_{1}}\left(\tau_{b}^{*}-\tau_{c r}^{*}\right)_{+}^{m_{2}-1} \operatorname{sgn}\left(\tau_{b}\right) Q,
$$

where

$$
\alpha=\frac{K_{d}}{K_{e} d_{s}} .
$$

That is,

$$
\widetilde{q}_{b}\left(h, h u, z_{m}\right)=z_{m} v_{b}
$$

with

$$
v_{b}=\alpha c\left(\tau_{b}^{*}\right)^{m_{1}}\left(\tau_{b}^{*}-\tau_{c r}^{*}\right)_{+}^{m_{2}-1} \operatorname{sgn}\left(\tau_{b}\right) Q .
$$




\subsection{Properties}

Theorem 3.1. The proposed formula (17) has the following properties:

i) The formula depends explicitly on $z_{m}$ and the continuity equation preserves the sediment mass.

ii) It coincides with a classical solid transport discharge in the case of a quasi-uniform regime.

Proof.

i) From (4), the conservation of sediment mass is obvious as whenever $z_{m}=0$ we have that the solid flux is 0 which means that initial sediment mass is preserved in situations like the one described in Figure 3.

ii) In a quasi-uniform regime, the deposition rate equals the erosion rate $\left(\dot{z}_{d}=\dot{z}_{e}\right)$, then from (14) and (16), we have that

$$
\frac{z_{m}(1-\varphi)}{d_{s}}=\frac{K_{e}}{K_{d}}\left(\tau_{b}^{*}-\tau_{c r}^{*}\right)_{+}
$$

Note that $z_{m}(1-\varphi) / d_{s}$ represents a number of particles. Thus, whenever erosion and the deposition rates are equal, we may simplify the solid transport discharge (5),

$$
\widehat{q_{b}}=z_{m} v_{b}=\frac{K_{e} d_{s}}{K_{d}} \frac{v_{b}}{(1-\varphi)}\left(\tau_{b}^{*}-\tau_{c r}^{*}\right)_{+} .
$$

Replacing $\alpha$ and $v_{b}$ by their respective values (18) and (20), we recover equation (13):

$$
\widehat{q_{b}}=\frac{K_{e} d_{s}}{K_{d}} \frac{K_{d}}{K_{e} d_{s}} \frac{c}{(1-\varphi)}\left(\tau_{b}^{*}\right)^{m_{1}}\left(\tau_{b}^{*}-\tau_{c r}^{*}\right)_{+}^{m_{2}} \operatorname{sgn}\left(\tau_{b}\right) Q=q_{b} .
$$

In this case we obtain a solid transport discharge that can be written without dependence on $z_{m}$.

Remark 3.1. - In the framework of Shallow Water equations, $v_{b}$ can be defined in terms of $\sqrt{\tau_{b}^{*}}$. Note that if we approximate $v_{b}$ in terms of $\sqrt{\left(\tau_{b}^{*}-\tau_{c r}^{*}\right)_{+}}$then we obtain that $q_{b}$ is defined in terms of $\left(\tau^{*}-\tau_{c r}^{*}\right)^{3 / 2}$ corresponding, for example, to the Meyer-Peter $\&$ Müller or FernándezLuque and Van Beek formulae.

- To obtain (22) we have used the relation (21). Actually, the relation between the number of particles of the moving layer and $\left(\tau_{b}^{*}-\tau_{c r}^{*}\right)_{+}$is used in the deduction of some of the most known classical formulae for the solid transport discharge. Bagnold obtained such a linear relation by studying the momentum transfer due to the interaction of the particles with the fixed bed (see [23], [5]). At the fixed bed the fluid shear stress is reduced to the threshold value $\tau_{c r}$. That is, the momentum transfer within the moving layer $z_{m}$ is $\left(\tau_{b}^{*}-\tau_{c r}^{*}\right)_{+}$. That implies a limitation in the erosion rate. Consequently, a relation similar to (21) can be obtained. Moreover, such a linear relation has been also observed experimentally by Fernández-Luque and Van Beek (see [20]). 
Remark 3.2. The solid transport discharge depends on the hydrodynamical variables only through the shear stress or the Shields parameter. Thus, in what follows, it will be useful to rewrite

$$
q_{b} \equiv \phi\left(\tau_{b}^{*}\right) \frac{\operatorname{sgn}\left(\tau_{b}\right)}{1-\varphi} Q
$$

where

$$
\phi\left(\tau_{b}^{*}\right)=c\left(\tau_{b}^{*}\right)^{m_{1}}\left(\tau_{b}^{*}-\tau_{c r}^{*}\right)^{m_{2}}
$$

and

$$
\widetilde{q_{b}} \equiv z_{m} v_{b}
$$

with

$$
v_{b} \equiv \alpha \frac{\phi\left(\tau_{b}^{*}\right)}{\tau_{b}^{*}-\tau_{c r}^{*}} \operatorname{sgn}\left(\tau_{b}\right) Q
$$

To sum up, we have that the right hand side of the last two equations of (2) can be considered to be zero for uniform flows where the rate of erosion and deposition are equal. In this case we may consider a solid transport discharge formula that is independent of $z_{m}$ (classical bedload transport flux). Consequently, if the rate of erosion and deposition are not equal, in the case of a non-uniform flow, definition of a solid transport discharge independent of $z_{m}$ cannot be valid. For example, this is the case that we have shown in Figure 3, and that implies the non conservation of the sediment mass when classical formulae for $\widehat{q_{b}}$, independent of $z_{m}$, are applied.

\section{Eigenvalues of Exner system for modified bedload transport formulae}

We are interested in the study of the eigenvalues of system (2). The PDE system (2) can be written in vectorial form as

$$
\partial_{t} \widetilde{W}+\partial_{x} \widetilde{F}(W)=\widetilde{B}(W) \partial_{x} \widetilde{W}+\widetilde{S}(W) \partial_{x} z_{r}+\widetilde{G}
$$

where

$$
W=\left(\begin{array}{c}
h \\
q \\
z_{m}
\end{array}\right) \in \mathbb{R}^{3}, \quad \widetilde{W}=\left(\frac{W}{z_{f}}\right) \in \mathbb{R}^{4}
$$

is the state vector in conservative form, where $q=h u$, and

$$
\begin{gathered}
\tilde{F}(W)=\left(\frac{F(W)}{0}\right), \quad F(W)=\left(\begin{array}{c}
h u \\
h u^{2}+\frac{g}{2} h^{2} \\
z_{m} v_{b}(h, h u)
\end{array}\right), \quad \widetilde{B}(W)=\left(\begin{array}{c}
B(W) \mid \\
\hline 0
\end{array}\right) \\
B(W)=\left(\begin{array}{ccc}
0 & 0 & 0 \\
0 & 0 & -g h \\
0 & 0 & 0
\end{array}\right), \quad S(W)=\left(\begin{array}{c}
0 \\
-g h \\
0
\end{array}\right), \quad \widetilde{S}(W)=\left(\frac{S(W)}{0}\right),
\end{gathered}
$$




$$
\widetilde{G}=\left(\frac{G}{-\dot{z}_{e}+\dot{z}_{d}}\right), \quad G=\left(\begin{array}{c}
0 \\
0 \\
\dot{z}_{e}-\dot{z}_{d}
\end{array}\right) .
$$

The definition of $v_{b}(h, q)$ can be considered following the proposed model by (20). Let us remark that with these notations system (23) can be rewritten as

$$
\left\{\begin{array}{l}
\partial_{t} W+\partial_{x} F(W)=B(W) \partial_{x} W+S(W) \partial_{x}\left(z_{r}+z_{f}\right)+G \\
\partial_{t} z_{f}=-\dot{z}_{e}+\dot{z}_{d}
\end{array}\right.
$$

System (23) can also be written in quasi-linear form as

$$
\partial_{t} \widetilde{W}+\widetilde{A}(W) \partial_{x} \widetilde{W}=\widetilde{S}(W) \partial_{x} z_{r}+\widetilde{G}
$$

where $\widetilde{A}(W)=D_{\widetilde{W}} \widetilde{F}-\widetilde{B}(W)$ is the matrix of transport coefficients. More explicitly, taking into account that $v_{b}=v_{b}\left(\tau_{b}\right)$ with $\tau_{b} \equiv \tau_{b}(h, q)$, we have

$$
\widetilde{A}(W)=\left(\begin{array}{c|c}
A(W) & S(W) \\
\hline 0 & 0
\end{array}\right)
$$

where $A(W)=D_{W} F(W)-B(W)$,

$$
A(W)=\left(\begin{array}{ccc}
0 & 1 & 0 \\
g h-u^{2} & 2 u & g h \\
z_{m} \frac{\partial v_{b}}{\partial h} & z_{m} \frac{\partial v_{b}}{\partial q} & v_{b}
\end{array}\right) .
$$

An important property of such systems is hyperbolicity [24] which requires that the matrix $\widetilde{A}(W)$ is $\mathbf{R}$ diagonalizable (or strictly hyperbolic when eigenvalues are distinct). Given the definition of $\widetilde{A}(W)$, we are concerned whether $A(W)$ is diagonalizable.

In what follows, we shall consider a classical bedload transport flux $q_{b}$ in the form (13) and we shall assume that $q_{b}$ satisfies the following hypothesis:

- The solid transport flux is an increasing function of the discharge

$$
\frac{\partial q_{b}}{\partial q} \geq 0
$$

- There exists a constant $k>0$ such that

$$
\frac{\partial q_{b}}{\partial h}=-k \frac{q}{h} \frac{\partial q_{b}}{\partial q}
$$

Remark 4.1. 
- (H1) is equivalent to $\frac{\partial \tau_{b}}{\partial q} \geq 0$

To show this, remark that from (6) we have

$$
\tau_{b}^{*}=\beta\left|\tau_{b}\right|,
$$

where $\beta=\left(\left(\rho_{s}-\rho\right) g d_{s}\right)^{-1}$ and

$$
\partial \tau_{b}^{*}=\beta \operatorname{sgn}\left(\tau_{b}\right) \partial \tau_{b} .
$$

Now, considering the case $\tau_{b}^{*}>\tau_{c r}^{*}$, (the other case being trivial), we have

$$
\frac{\partial q_{b}}{\partial q}=k\left(m_{1}\left(\tau_{b}^{*}-\tau_{c r}^{*}\right)+m_{2} \tau_{b}^{*}\right)\left(\tau_{b}^{*}\right)^{m_{1}-1}\left(\tau_{b}^{*}-\tau_{c r}\right)^{m_{2}-1} \beta \frac{\partial \tau_{b}}{\partial q},
$$

and the result follows.

- (H2) is equivalent to

$$
\frac{\partial \tau_{b}}{\partial h}=-k \frac{q}{h} \frac{\partial \tau_{b}}{\partial q} .
$$

This can be easily shown by comparing (26) with

$$
\frac{\partial q_{b}}{\partial h}=k\left(m_{1}\left(\tau_{b}^{*}-\tau_{c r}^{*}\right)+m_{2} \tau^{*}\right)\left(\tau_{b}^{*}\right)^{m_{1}-1}\left(\tau_{b}^{*}-\tau_{c r}\right)^{m_{2}-1} \beta \frac{\partial \tau_{b}}{\partial h} .
$$

- In [25], it was shown that classical formulae satisfy in general the relation

$$
\frac{\partial q_{b}}{\partial h}=-k \frac{q}{h} \frac{\partial q_{b}}{\partial q}
$$

where $k$ is a given positive constant. For instance, for Grass model [13] we have $k=1$ and for Meyer-PeterËMüller [14] we have $k=7 / 6$.

Proposition 4.1. Consider any classical flux $q_{b}$ in the form (13) that satisfies (H1) and (H2), then $v_{b}$ given by (20) satisfies

- $\frac{\partial v_{b}}{\partial q} \geq 0$

- $\frac{\partial v_{b}}{\partial h}=-k \frac{q}{h} \frac{\partial v_{b}}{\partial q}$

Proof. From Remark 3.2, a simple calculation shows

$$
\partial v_{b}=\alpha \frac{\phi^{\prime}\left(\tau_{b}^{*}\right)\left(\tau_{b}^{*}-\tau_{c r}^{*}\right)-\phi\left(\tau_{b}^{*}\right)}{\left(\tau_{b}^{*}-\tau_{c r}^{*}\right)^{2}} \beta \partial \tau_{b}^{*}
$$

and from (H1) - (H2) the result follows. 


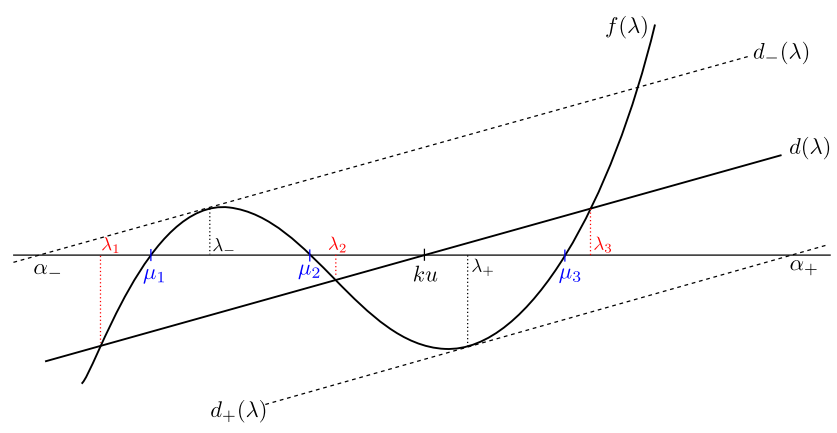

Figure 4: Hyperbolicity of Exner system

As a consequence, the matrix $A(W)$ defined by $(25)$ can be written as

$$
A(W)=\left(\begin{array}{ccc}
0 & 1 & 0 \\
g h-u^{2} & 2 u & g h \\
-k u b & b & v_{b}
\end{array}\right),
$$

where $b=z_{m} \frac{\partial v_{b}}{\partial q}$.

The characteristic polynomial of $A(W)$ can then be written as

$$
\begin{aligned}
p_{A}(\lambda) & =\left(v_{b}-\lambda\right)\left|\begin{array}{cc}
-\lambda & 1 \\
-u^{2}+g h & 2 u-\lambda
\end{array}\right|-g h\left|\begin{array}{cc}
-\lambda & 1 \\
-k u b & b
\end{array}\right| \\
& \left.=\left(v_{b}-\lambda\right)\left[(u-\lambda)^{2}-g h\right)\right]+g h b(\lambda-k u) .
\end{aligned}
$$

In what follows, let us denote by

$$
\left\{\mu_{1}, \mu_{2}, \mu_{3}\right\}=\left\{v_{b}, u \pm \sqrt{g h}\right\} \quad \text { with } \quad \mu_{1}<\mu_{2}<\mu_{3}
$$

System (23) is thus strictly hyperbolic if and only if $p_{A}(\lambda)$ has three different solutions noted by $\lambda_{1}<\lambda_{2}<\lambda_{3}$. In other words if the curve $\left.f(\lambda)=\left(\lambda-v_{b}\right)\left[(u-\lambda)^{2}-g h\right)\right]$ and the line $d(\lambda)=g h b(\lambda-k u)$ have three distinct points of intersection (see Figure 4). We have the following result:

Proposition 4.2. Consider system (23) with (13) satisfying (H1) - (H2).

For a given state $(h, q)$, the system is strictly hyperbolic if and only if

$$
\alpha_{-}<k u<\alpha_{+}
$$

where $\alpha_{ \pm}$will be defined later by expression (57). More explicitly,

- In the case $k=1$, the system is always strictly hyperbolic.

- In the case $k=7 / 6$, a sufficient condition for system (23) to be strictly hyperbolic is

$$
|u|<6 \sqrt{g h}
$$


Let us remark that we obtain a similar result to the case where bedload sediment transport formula does not depend on $z_{m}$, as it is shown in [25]. The proof is not exactly the same but can be done by following similar steps as in [25]. The proof is included in Appendix A for the sake of completeness.

We are interested in classifying the different roots of the polynomial $p_{A}(\lambda)$. More explicitly, in the case of classical bedload transport flux, it is a known fact (see [25] and references therein) that we have always two eigenvalues of the same sign and a third one of opposite sign. We intend to show that the behaviour with the modified bedload flux (17) is quite different. Assume that the system is strictly hyperbolic and denote by $\lambda_{1}<\lambda_{2}<\lambda_{3}$ the three roots of $p_{A}(\lambda)$.

Remark that (29) may be written in the form

$$
p_{A}(\lambda)=-\lambda^{3}+a_{2} \lambda^{2}+a_{1} \lambda+a_{0},
$$

where $a_{0}, a_{1}, a_{2}$ are the corresponding coefficients of the polynomial. In what interests us, we remark that

$$
a_{0}=v_{b}\left(u^{2}-g h\right)-k b h u g .
$$

Moreover, we also have $p_{A}(\lambda)=-\left(\lambda-\lambda_{1}\right)\left(\lambda-\lambda_{2}\right)\left(\lambda-\lambda_{3}\right)$ and $a_{0}=\lambda_{1} \lambda_{2} \lambda_{3}$.

We have 4 different cases:

Case 1: $0<\mu_{1}$. Then two possibilities arise (see Figure 5)

- $a_{0}<0$. We have two positive eigenvalues and one negative (Figure 5(a))

- $a_{0}>0$. We have three positive eigenvalues (Figure 5(b)))

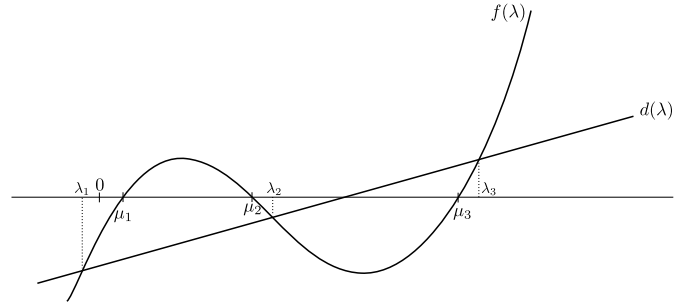

(a)

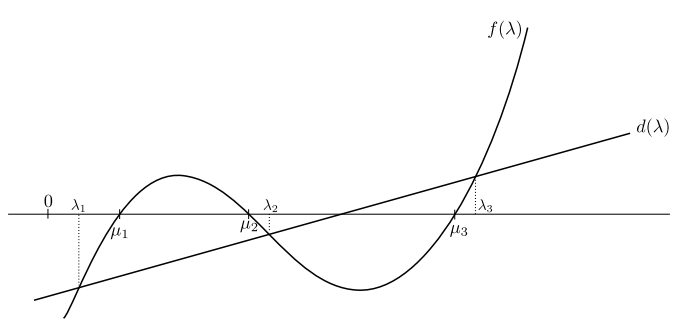

(b)

Figure 5: Case $0<\mu_{1}$

Remark that $a_{0}>0$ is equivalent to

$$
1<\frac{v_{b}\left(u^{2}-g h\right)}{k b h u g}=\frac{v_{b}\left(u^{2}-g h\right)}{k z_{m} \frac{\partial v_{b}}{\partial(h u)} h u g} .
$$




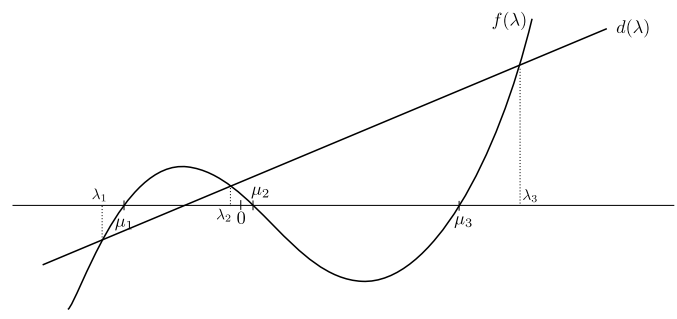

(a)

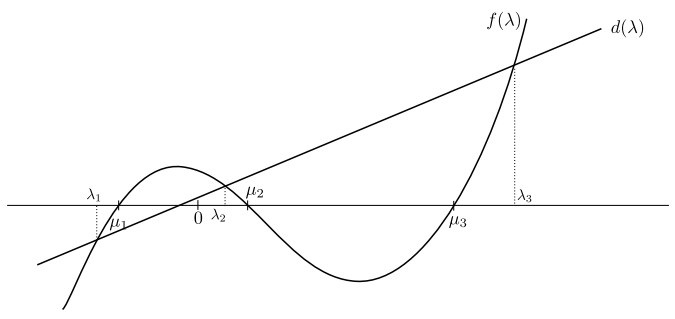

(b)

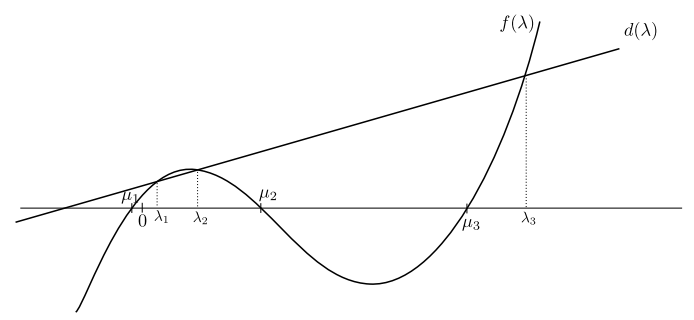

(c)

Figure 6: Case $\mu_{1}<0<\mu_{2}<\mu_{3}$

As $\mu_{1}>0$, we are in a supercritical regime $u^{2}-g h>0$. Moreover, $v_{b} /(h u)>0$ and $\frac{\partial v_{b}}{\partial(h u)}>0$. Thus

$$
z_{m}<\frac{v_{b}\left(u^{2}-g h\right)}{k \frac{\partial v_{b}}{\partial(h u)} h u g}
$$

is satisfied if and only if $z_{m}$ is small enough.

Case 2: $\mu_{1}<0<\mu_{2}<\mu_{3}$. Then we have three possibilities:

- $\lambda_{1}<\lambda_{2}<0<\lambda_{3}$ (Figure 6(a))

- $\lambda_{1}<0<\lambda_{2}<\lambda_{3}$ (Figure 6(b))

- $0<\lambda_{1}<\lambda_{2}<\lambda_{3}$ (Figure 6(c))

But now remark that $\operatorname{sgn}\left(v_{b}\right)=\operatorname{sgn}(u)$, and as $\mu_{1}<0<\mu_{2}<\mu_{3}$, we have necessarily $u>0$ (otherwise we would have $v_{b}<0$ and $u-\sqrt{g h}<0$ ). This means that we are in a subcritical regime $u^{2}-g h<0$,

$$
a_{0}=v_{b}\left(u^{2}-g h\right)-k b h u g<0,
$$

and $\operatorname{sgn}\left(a_{0}\right)=\operatorname{sgn}\left(\lambda_{1} \lambda_{2}\right)$. As a consequence, the only possible case is $\lambda_{1}<0$ and $\lambda_{2}, \lambda_{3}>0$.

Case 3: $\mu_{1}<\mu_{2}<0<\mu_{3}$ Then we have three possibilities (Figure 7)

- $\lambda_{1}<\lambda_{2}<\lambda_{3}<0$ (Figure $7(\mathrm{a})$ )

- $\lambda_{1}<\lambda_{2}<0<\lambda_{3}$ (Figure 7(b)) 


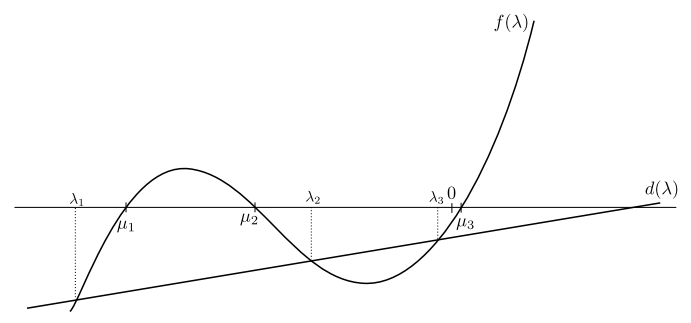

(a)

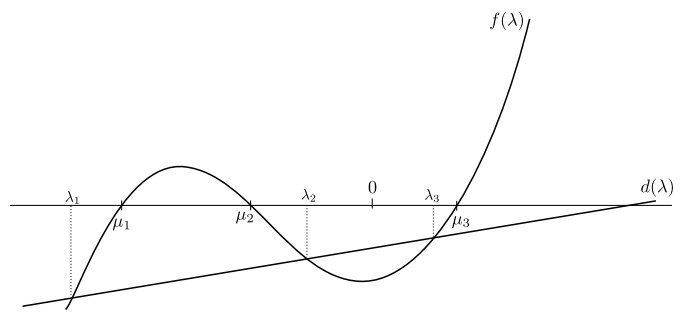

(b)

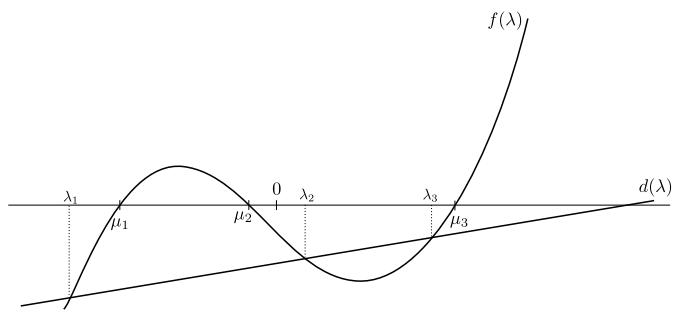

(c)

Figure 7: Case $\mu_{1}<\mu_{2}<0<\mu_{3}$

- $\lambda_{1}<0<\lambda_{2}<\lambda_{3}$ (Figure $7(\mathrm{c})$ )

As $\operatorname{sgn}\left(v_{b}\right)=\operatorname{sgn}(u)$ we should have $u<0$, otherwise we would have $u+\sqrt{g h}>0$ and $v_{b}>0$. This means we are in a subcritical region $u^{2}-g h<0$. Thus

$$
a_{0}=v_{b}\left(u^{2}-g h\right)-k b h u g>0,
$$

and we have $\operatorname{sgn}\left(a_{0}\right)=-\operatorname{sgn}\left(\lambda_{2} \lambda_{3}\right)$. As a consequence the only possible case is $\lambda_{1}, \lambda_{2}<0$ and $\lambda_{3}>0$.

Case 4: $\mu_{1}<\mu_{2}<\mu_{3}<0$ Then two possibilities arise (see Figure 8)

- $a_{0}<0$. We have two negative eigenvalues and one positive (Figure 5(a))

- $a_{0}>0$. We have three negative eigenvalues (Figure $\left.5(\mathrm{~b})\right)$ )

Remark that $a_{0}>0$ is equivalent to

$$
1<\frac{v_{b}\left(u^{2}-g h\right)}{k b h u g}=\frac{v_{b}\left(u^{2}-g h\right)}{k z_{m} \frac{\partial v_{b}}{\partial(h u)} h u g} .
$$

In this case, we have $u^{2}-g h>0$ (supercritical regime) and $a_{0}>0$ is equivalent to

$$
\frac{v_{b}\left(u^{2}-g h\right)}{k \frac{\partial v_{b}}{\partial(h u)} h u g}>z_{m},
$$

which is satisfied if and only if $z_{m}$ is small enough. 


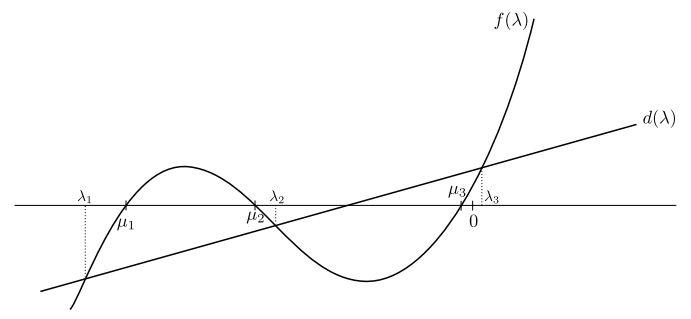

(a)

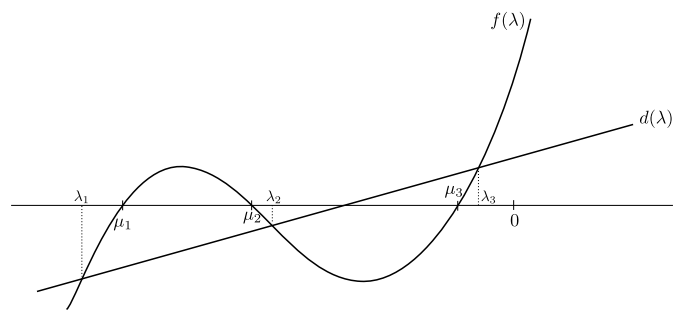

(b)

Figure 8: Case $\mu_{1}<\mu_{2}<\mu_{3}<0$

To summarize the results:

- In the subcritical case $\left(u^{2}-g h<0\right)$, we have two eigenvalues of the same sign and one of opposite sign.

- In the supercritical case $\left(u^{2}-g h>0\right)$, we have two possibilities:

- Three eigenvalues of the same sign,

- Two eigenvalues of the same sign and one of opposite sign.

Remark that the supercritical region presents a major difference compared to classical bedload transport formulae. In the classical case, we always have two eigenvalues of the same sign and one of different sign, but here there is a new possibility of having three eigenvalues of the same sign due to the new solid transport discharge.

In this paper, we have presented two forms to obtain a solid transport discharge depending on $z_{m}$. The first one was proposed by Fowler et al. in [17] as an extension of the Meyer-Peter \& Müller formula (see Section 1). Although the idea can be easily extended for other classical models. The second one has been proposed in Section 3. If we consider a classical model that can be written as

$$
q_{b}(h, u)=c\left(\tau_{b}^{*}\right)^{m_{1}}\left(\tau_{b}^{*}-\tau_{c r}^{*}\right)_{+}^{m_{2}},
$$

then, we can resume both models as follows,

$$
\widetilde{q}\left(h, u, z_{b}\right)=z_{m} v_{b},
$$

with $v_{b}$ defined as follows:

- In the case of the extension proposed by Fowler et al. we have:

$$
v_{b}=c_{1}\left(\tau_{b}^{*}\right)^{m_{1}}\left(\tau_{b}^{*}-\tau_{c r}^{*}\right)_{+}^{m_{2}} .
$$

- In the case of the extension proposed in Section 3 we have:

$$
v_{b}=c_{2}\left(\tau_{b}^{*}\right)^{m_{1}}\left(\tau_{b}^{*}-\tau_{c r}^{*}\right)_{+}^{m_{2}-1},
$$


being $c_{1}$ and $c_{2}$ two different constant values. The value of these constants does not have any influence on the condition

$$
z_{m}<\frac{v_{b}\left(u^{2}-g h\right)}{k \frac{\partial v_{b}}{\partial(h u)} h u g} .
$$

In order to study the influence of the considered model in condition (30), it is enough to study the ratio $v_{b} / \frac{\partial v_{b}}{\partial(h u)}$. When we consider the extension proposed by Fowler et al. we obtain,

$$
\frac{v_{b}}{\frac{\partial v_{b}}{\partial(h u)}}=\frac{1}{2} \frac{h u}{m_{1}+m_{2} \frac{\tau_{b}^{*}}{\left(\tau_{b}^{*}-\tau_{c r}^{*}\right)_{+}}} .
$$

For the model proposed in Section 3 we obtain the same result but by substituting $m_{2}$ by $m_{2}-1$. Consequently, if we consider the Meyer-Peter \& Müller formula, corresponding to $m_{1}=0, m_{2}=3 / 2$, we obtain that the maximum value of $z_{m}$ verifying condition (30) for the case of the model proposed by Fowler et al. is 3 times smaller than for the case of the model proposed in Section 3.

\section{Numerical results}

\subsection{Numerical approach by finite volume methods}

As usual, we consider a set of computing cells $I_{i}=\left[x_{i-1 / 2}, x_{i+1 / 2}\right], i \in \mathbb{Z}$. We shall assume that these cells have a constant size $\triangle x$ and that $x_{i+1 / 2}=i \triangle x$. The point $x_{i}=(i-1 / 2) \triangle x$ is the center of the cell $I_{i}$. Let $\triangle t$ be the time step and $t^{n}=n \triangle t$.

We denote by $W_{i}^{n}=\left(h_{i}^{n}, q_{i}^{n}, z_{m, i}^{n}\right)^{T}$ the approximation of the cell averages of the exact solution

$$
W_{i}^{n} \cong \frac{1}{\triangle x} \int_{x_{i-1 / 2}}^{x_{i+1 / 2}} W\left(x, t^{n}\right) d x
$$

and

$$
H_{i}^{n}=z_{f, i}^{n}+z_{r, i}, \quad \text { with } \quad z_{f, i}^{n} \cong \frac{1}{\triangle x} \int_{x_{i-1 / 2}}^{x_{i+1 / 2}} z_{f}\left(x, t^{n}\right) d x, \quad z_{r, i} \cong \frac{1}{\triangle x} \int_{x_{i-1 / 2}}^{x_{i+1 / 2}} z_{r}(x) d x
$$

Following the ideas presented in [26], we propose a two-step numerical scheme by solving first the pure hydrodynamical part and then by treating the transfer terms between the static layer and the rolling particles layer in a second step.

Let us suppose that the values $W_{i}^{n}$ and $H_{i}^{n}$ are known. In order to advance in time we proceed as follows:

- First Step. We solve first the hyperbolic non-conservative system (2) where we neglect the erosion and deposition terms which will be treated in the second step. To solve the system, we propose here a scheme based on the theory of path-conservative numerical schemes introduced in [27]. In particular, we propose here a Roe scheme based on a Roe linearization of the matrix $A(W)$ in the sense introduced in $[28,29,30]$. For the sake of completeness, we give here the main ideas of the scheme. 
Given a family of paths $\Phi$, a matrix function $A_{\Phi}: \Omega \times \Omega \rightarrow \mathcal{M}_{N}(\mathbb{R})$ is called a Roe linearization if it satisfies:

- for any $W_{l}, W_{r} \in \Omega, A_{\Phi}\left(W_{l}, W_{r}\right)$ has $N$ real eigenvalues;

- $A_{\Phi}(W, W)=A(W)$, for all $W \in \Omega$;

- for any $W_{l}, W_{r} \in \Omega$ :

$$
A_{\Phi}\left(W_{l}, W_{r}\right)\left(W_{r}-W_{l}\right)=\int_{0}^{1} A\left(\Phi\left(s ; W_{l}, W_{r}\right)\right) \partial_{s} \Phi\left(s ; W_{l}, W_{r}\right) d s .
$$

The simplest choice of paths is the family of segments $\Phi\left(s, W_{l}, W_{r}\right)=W_{l}+s\left(W_{r}-W_{l}\right)$. Once a Roe linearization has been selected, a path-conservative Roe scheme can be defined as follows: We define $W_{i}^{n+1 / 2}=\left(h_{i}^{n+1 / 2}, q_{i}^{n+1 / 2}, z_{m, i}^{n+1 / 2}\right)^{T}$ as

$$
W_{i}^{n+1 / 2}=W_{i}^{n}-\frac{\Delta t}{\Delta x}\left(D_{i-1 / 2}^{n,+}+D_{i+1 / 2}^{n,-}\right),
$$

where $D_{i+1 / 2}^{n, \pm}=D\left(W_{i}^{n}, W_{i+1}^{n}, H_{i}^{n}, H_{i+1}^{n}\right)^{ \pm}$, being

$$
\begin{gathered}
D\left(W_{i}, W_{i+1}, H_{i}, H_{i+1}\right)^{ \pm}=\frac{1}{2}\left(I \pm \operatorname{sgn}\left(A_{i+1 / 2}\right)\right) \\
\left(F\left(W_{i+1}\right)-F\left(W_{i}\right)+B_{i+1 / 2}\left(W_{i+1}-W_{i}\right)\right. \\
\left.+S_{i+1 / 2}\left(H_{i+1}-H_{i}\right)\right) .
\end{gathered}
$$

where $I$ is the identity matrix, $A_{i+1 / 2}=A_{\Phi}\left(W_{i}, W_{i+1}\right), \operatorname{sgn}\left(A_{i+1 / 2}\right)$ is the matrix sign of $A_{i+1 / 2}$ and

$$
B_{i+1 / 2}=B\left(\frac{W_{i}+W_{i+1}}{2}\right), \quad S_{i+1 / 2}=S\left(\frac{W_{i}+W_{i+1}}{2}\right) .
$$

As it was stated in [9] it is possible to write an exact Roe matrix for the Grass model for the family path of segments. Nevertheless, it is not possible or it is very costly to write a exact Roe matrix for other models, such as Meyer-Peter\&Müller or some other models considered in this work. So, in practice the following approximation can be used

$$
A_{i+1 / 2}=\left(\begin{array}{ccc}
0 & 1 & 0 \\
g h_{i+1 / 2}-u_{i+1 / 2}^{2} & 2 u_{i+1 / 2} & g h_{i+1 / 2} \\
-k u_{i+1 / 2} b_{i+1 / 2} & b_{i+1 / 2} & v_{b, i+1 / 2}
\end{array}\right) \text {, }
$$

where we assume

$$
\frac{\partial q_{b}}{\partial h}=-k \frac{q}{h} \frac{\partial q_{b}}{\partial q}
$$

that is a Roe matrix in the form (28). In particular, the usual definitions

$$
h_{i+1 / 2}=\frac{h_{i}+h_{i+1}}{2}, \quad u_{i+1 / 2}=\frac{\sqrt{h_{i}} u_{i}+\sqrt{h_{i+1}} u_{i+1}}{\sqrt{h_{i}}+\sqrt{h_{i+1}}},
$$

are taken, which corresponds to the choice of segments as the path connecting two different states. See $[28,29,30]$ for further details. 
The term $b_{i+1 / 2}$ is approximated by

$$
b_{i+1 / 2}=\frac{z_{m, i}+z_{m, i+1}}{2} \frac{\partial q_{b}}{\partial(h u)}\left(h_{i+1 / 2}, h_{i+1 / 2} u_{i+1 / 2}\right) .
$$

An other possibility in approaching the matrix $A_{i+1 / 2}$ is to use the technique proposed in $[31,32,33]$. In order to compute an approximation of the Roe linearization for the family of segments we should have

$$
A_{i+1 / 2}=\int_{0}^{1} A\left(\Phi\left(s, W_{i}, W_{i+1}\right)\right) d s .
$$

The right hand side of (34) can be approximated directly via a Gaussian quadrature rule of suitably high order of accuracy. This purely numerical procedure does not require the explicit computation of the Roe averages. Therefore, using a Gaussian quadrature rule with $M$ points on the unit interval $[0,1]$, weights $\omega_{i}$ and positions $\mu_{i}$ respectively, we compute an approximation of $A_{i+1 / 2}$ by

$$
A_{i+1 / 2} \approx \sum_{j=1}^{M} \omega_{i} A\left(\Phi\left(\mu_{i}, W_{i}, W_{i+1}\right)\right) .
$$

\section{- Second step.}

Next, we take into account the erosion and deposition rates.

We define

$$
W_{i}^{n+1}=\left(h_{i}^{n+1 / 2}, q_{i}^{n+1 / 2}, z_{m, i}^{n+1}\right)^{T}, \quad H_{i}^{n+1}=z_{f, i}^{n+1}+z_{r, i},
$$

where

$$
\begin{aligned}
& \text { where }\left\{\begin{array}{l}
z_{m, i}^{n+1}=z_{m, i}^{n}+\Delta t\left(\dot{z}_{e, i}^{n+1 / 2}-\dot{z}_{d, i}^{n+1 / 2}\right), \\
z_{f, i}^{n+1}=z_{f, i}^{n}+\Delta t\left(-\dot{z}_{e, i}^{n+1 / 2}+\dot{z}_{d, i}^{n+1 / 2}\right),
\end{array}\right. \\
& \text { and } \dot{z}_{e, i}^{n+1 / 2}=\dot{z}_{e, i}\left(W_{i}^{n+1 / 2}\right), \dot{z}_{d, i}^{n+1 / 2}=\dot{z}_{d, i}\left(W_{i}^{n+1 / 2}\right) .
\end{aligned}
$$

\subsubsection{High order extension}

In order to extend the numerical scheme to high order, let us consider a state reconstruction operator $P^{t}$, that is, an operator that associates, to a given sequence $\left\{W_{i}(t)\right\}$, two new sequences $\left\{W_{i+1 / 2}^{-}(t)\right\}$, $\left\{W_{i+1 / 2}^{+}(t)\right\}$ in such a way that, whenever

$$
W_{i}(t)=\frac{1}{\Delta x} \int_{I_{i}} W(x, t) d x, \forall i \in \mathbb{Z}
$$

for some regular function $W$, then

$$
W_{i+1 / 2}^{ \pm}(t)=W\left(x_{i+1 / 2}, t\right)+O\left(\Delta x^{p}\right), \forall i \in \mathbb{Z}
$$

For conservative hyperbolic systems it is enough with these reconstructions, nevertheless for nonconservative terms it is necessary to know the state reconstruction over the interval $I_{i}$. 
Then, firstly, over each control volume $I_{i}$, at each instant $t>0$, we define regular functions $\widetilde{P}_{i}$ and $P_{i}^{z_{r}}$ such that

$$
\begin{gathered}
\lim _{x \rightarrow x_{i-1 / 2}^{+}} \widetilde{P}_{i}(x)=\widetilde{W}_{i-1 / 2}^{+}(t), \quad \lim _{x \rightarrow x_{i+1 / 2}^{-}} \widetilde{P}_{i}(x)=\widetilde{W}_{i+1 / 2}^{-}(t), \\
\lim _{x \rightarrow x_{i-1 / 2}^{+}} P_{i}^{z_{r}}(x)=\left(z_{r}\right)_{i-1 / 2}^{+}(t), \quad \lim _{x \rightarrow x_{i+1 / 2}^{-}} P_{i}^{z_{r}}(x)=\left(z_{r}\right)_{i+1 / 2}^{-}(t) .
\end{gathered}
$$

We shall denote by $P_{i}(x)$ and $P_{i}^{z_{f}}(x)$ the first 3 components and the last component of $\widetilde{P}_{i}(x)$ respectively and $P_{i}^{H}=P_{i}^{z_{f}}+P_{i}^{z_{r}}$

Then, following [34], we propose the numerical scheme:

$$
\left\{\begin{aligned}
\frac{d}{d t} W_{i}= & -\frac{1}{\Delta x}\left(D_{i-1 / 2}^{+}+D_{i+1 / 2}^{-}\right. \\
& \left.+\int_{I_{i}} A\left(P_{i}(x)\right) \frac{d}{d x} P_{i}(x) d x+\int_{I_{i}} S\left(P_{i}(x)\right) \frac{d}{d x} P_{i}^{H}(x) d x\right)+G\left(W_{i}\right), \\
\frac{d}{d t}\left(z_{f}\right)_{i}= & -\dot{z}_{e}+\dot{z}_{d}
\end{aligned}\right.
$$

where

$$
\begin{aligned}
D_{i+1 / 2}^{ \pm}=D\left(\widetilde{W}_{i+1 / 2}^{-}, \widetilde{W}_{i+1 / 2}^{+}\right)=\frac{1}{2}( & \left.I \pm \operatorname{sgn}\left(A_{i+1 / 2}\right)\right)\left(F\left(W_{i+1 / 2}^{+}\right)-F\left(W_{i-1 / 2}^{-}\right)\right. \\
& \left.+B_{i+1 / 2}\left(W_{i+1 / 2}^{+}-W_{i+1 / 2}^{-}\right)+S_{i+1 / 2}\left(H_{i+1 / 2}^{+}-H_{i+1 / 2}^{-}\right)\right)
\end{aligned}
$$

and now $B_{i+1 / 2}, S_{i+1 / 2}, A_{i+1 / 2}$ are defined in a similar way as in (32) - (33) but using the reconstructed states $W_{i+1 / 2}^{ \pm}$.

For the time evolution, we use a high order TVD Runge-Kutta as the one proposed in [35].

\subsubsection{Application to the 2D case}

The $2 \mathrm{D}$ version of system (2) writes

$$
\left\{\begin{array}{l}
\partial_{t} h+\partial_{x}\left(h u^{x}\right)+\partial_{y}\left(h u^{y}\right)=0 \\
\partial_{t}\left(h u^{x}\right)+\partial_{x}\left(h\left(u^{x}\right)^{2}+g h^{2} / 2\right)+\partial_{y}\left(h u^{x} u^{y}\right)=-g h \partial_{x}\left(z_{m}+z_{f}+z_{r}\right), \\
\partial_{t}\left(h u^{y}\right)+\partial_{x}\left(h u^{x} u^{y}\right)+\partial_{y}\left(h\left(u^{y}\right)^{2}+g h^{2} / 2\right)=-g h \partial_{y}\left(z_{m}+z_{f}+z_{r}\right), \\
\partial_{t} z_{m}+\partial_{x} \widetilde{q}_{b}^{x}+\partial_{y} \widetilde{q}_{b}^{y}=\dot{z}_{e}-\dot{z}_{d} \\
\partial_{t} z_{f}=-\dot{z}_{e}+\dot{z}_{d}
\end{array}\right.
$$

where $\mathbf{u}=\left(u^{x}, u^{y}\right)$ are the components of the velocity vector and

$$
\widetilde{\mathbf{q}_{\mathbf{b}}}=\left({\widetilde{q_{b}}}^{x},{\widetilde{q_{b}}}^{y}\right)={\widetilde{q_{b}}}_{b}\left(h, h|\mathbf{u}|, z_{m}\right) \operatorname{sgn}(\mathbf{u}),
$$

where $\widetilde{q}_{b}\left(h, h u, z_{m}\right)$ is given by the $1 \mathrm{D}$ model $(17)$. Here we are using the notation

$$
|\mathbf{v}|=\sqrt{\left(v^{x}\right)^{2}+\left(v^{y}\right)^{2}}, \quad \operatorname{sgn}(\mathbf{v})=\frac{\mathbf{v}}{|\mathbf{v}|}, \quad \text { for any vector } \mathbf{v}=\left(v^{x}, v^{y}\right) \in \mathbb{R}^{2} .
$$


It is easy to define a numerical scheme for the $2 \mathrm{D}$ problem (40) from a $1 \mathrm{D}$ numerical scheme. The point is to follow the ideas presented in [36].

System (40) can be written in the form

$$
\partial_{t} \widetilde{W}+\partial_{x} \widetilde{F_{1}}(W)+\partial_{y} \widetilde{F_{2}}(W)=\widetilde{B_{1}}(W) \partial_{x} \widetilde{W}+\widetilde{B_{2}}(W) \partial_{y} \widetilde{W}+\widetilde{S_{1}}(W) \partial_{x} z_{r}+\widetilde{S_{2}}(W) \partial_{y} z_{r}+\widetilde{G}
$$

where $W=\left(h, h u^{x}, h u^{y}, z_{m}\right)^{T}$ is the state vector, $\widetilde{W}=\left(W^{T}, z_{f}\right)^{T}$,

$$
\begin{gathered}
F_{1}(W)=\left(h u^{x}, h\left(u^{x}\right)^{2}+1 / 2 g h^{2}, h u^{x} u^{y}, \widetilde{q}_{b}^{x}\right)^{T}, \\
F_{2}(W)=\left(h u^{y}, h u^{x} u^{y}, h\left(u^{y}\right)^{2}+1 / 2 g h^{2},{\widetilde{q_{b}}}^{y}\right)^{T}, \\
\widetilde{F_{j}}(W)=\left(F_{j}(W)^{T}, 0\right)^{T}, \quad j=1,2, \\
\widetilde{B_{j}}(W)=\left(\begin{array}{c|c}
B_{j}(W) & S_{j}(W) \\
\hline 0 & 0
\end{array}\right), j=1,2, \quad B_{1}(W)=-g h E_{2,4}, \quad B_{2}(W)=-g h E_{3,4},
\end{gathered}
$$

where $E_{i j}$ denotes the canonical base of matrices of size $4 \times 4$ and

$$
\widetilde{S_{j}}(W)=\left(S_{j}(W)^{T}, 0\right), j=1,2, \quad S_{1}(W)=(0,-g h, 0,0)^{T}, \quad S_{2}(W)=(0,0,-g h, 0)^{T} .
$$

$\widetilde{G}$ denotes the erosion and deposition terms

$$
\widetilde{G}=\left(G^{T},-\dot{z}_{e}+\dot{z}_{d}\right)^{T}, \quad G=\left(0,0,0, \dot{z}_{e}-\dot{z}_{d}\right)^{T} .
$$

Equation (42) can also be written in the form given by

$$
\left\{\begin{array}{l}
\partial_{t} W+A_{1}(W) \partial_{x} W+A_{2}(W) \partial_{y} W=S_{1}(W) \partial_{x}\left(z_{f}+z_{r}\right)+S_{2}(W) \partial_{y}\left(z_{f}+z_{r}\right)+G, \\
\partial_{t} z_{f}=-\dot{z}_{e}+\dot{z}_{d},
\end{array}\right.
$$

where $A_{j}(W)=D_{W} F_{j}(W)-B_{j}(W)$ for $j=1,2$.

System (40) is invariant under rotations. More explicitly: for any unit vector $\vec{n}=\left(n_{1}, n_{2}\right)$, let us define the matrix

$$
T_{\vec{n}}=\left(\begin{array}{cccc}
1 & 0 & 0 & 0 \\
0 & n_{1} & n_{2} & 0 \\
0 & -n_{2} & n_{1} & 0 \\
0 & 0 & 0 & 1
\end{array}\right)
$$

and denote by $F_{\vec{n}}=n_{1} F_{1}+n_{2} F_{2}$ and $\mathcal{B}(W)=\left(B_{1}(W), B_{2}(W)\right)$. Then, we have

$$
\begin{gathered}
F_{\vec{n}}=T_{\vec{n}}^{-1} F_{1}\left(T_{\vec{n}} W\right), \\
T_{\vec{n}}(\mathcal{B}(W) \cdot \vec{n})=B_{1}\left(T_{\vec{n}} W\right),
\end{gathered}
$$

and system (42), without erosion/deposition terms, is written equivalently in the form

$$
\partial_{t}\left(T_{\vec{n}} W\right)+F_{1}\left(T_{\vec{n}} W\right)=B_{1}\left(T_{\vec{n}} W\right) \partial_{\vec{n}} W+T_{\vec{n}}\left(-\partial_{\vec{n}^{\perp}} F_{\vec{n}^{\perp}}(W)+\left(\mathcal{B}(W) \cdot \vec{n}^{\perp}\right) \partial_{\vec{n}^{\perp}} W\right) .
$$


The design of the numerical scheme is done by using (53) at each edge of a mesh, being $\vec{n}$ the normal vector to the edge. We begin by defining a mesh of the considered domain $\Omega$ into control volumes. We denote the volumes that define the mesh by $K_{i}$. Let $\mathcal{E}_{i j}$ denote the common edge between the volumes $K_{i}$ and $K_{j}$ and $\vec{n}_{i j}$ the unitary normal vector to $\mathcal{E}_{i j}$ pointing from $K_{i}$ to $K_{j}$. The structure of a $2 \mathrm{D}$ finite volume method is given by

$$
W_{i}^{n+1}=W_{i}^{n}-\frac{\Delta t}{\left|K_{i}\right|} \sum_{j \in K_{i}}\left|\mathcal{E}_{i j}\right| \Psi\left(W_{i}^{n}, W_{j}^{n}, \vec{n}_{i j}\right)+\frac{\Delta t}{2\left|K_{i}\right|} \sum_{j \in K_{i}}\left|\mathcal{E}_{i j}\right| \mathcal{B}_{\vec{n}_{i j}}\left(W_{j}^{n}-W_{i}^{n}\right)
$$

where $\boldsymbol{\Psi}\left(W_{i}^{n}, W_{j}^{n}, \vec{n}_{i j}\right)$ is an approximation of the normal flux associated to the edge $\mathcal{E}_{i j}$ and $\mathcal{B}_{\vec{n}_{i j}}$ is an approximation of $\mathcal{B}(W) \cdot \vec{n}_{i j}$. The 2 D numerical flux $\boldsymbol{\Psi}\left(W_{i}^{n}, W_{j}^{n}, \vec{n}_{i j}\right)$ can be computed by

$$
\Psi\left(W_{i}^{n}, W_{j}^{n}, \vec{n}_{i j}\right)=T_{\vec{n}_{i j}}^{-1} \Psi\left(T_{\vec{n}_{i j}} W_{i}, T_{\vec{n}_{i j}} W_{j}\right) .
$$

where $\Psi\left(W_{i}, W_{j}\right)$ is the numerical flux function of a $1 \mathrm{D}$ system, obtained from the $2 \mathrm{D}$ system by a projection over $\vec{n}_{i j}$. This can be defined following the ideas presented previously for the 1D case.

\subsection{Numerical tests}

We present in this section several numerical tests. We have performed some purely academic tests, in order to validate the code, but also some more realistic tests (e.g. dune in two dimensions), and a comparison with experimental data.

Unless otherwise specified, the numerical tests have been performed using a first order scheme with 800 finite volumes.

\subsubsection{Eigenvalues in a supercritical case}

In this section, we give examples to illustrate the sign of eigenvalues for supercritical flows. We proved in section 4 that in the classical case, we always have two eigenvalues of the same sign and one of different sign, but here there is a new possibility of having three eigenvalues of the same sign due to the new solid transport discharge.

The first results are given in Figures 9-10: we plot the eigenvalues for a supercritical case where both transport discharges have the same behavior, namely two eigenvalues of the same sign and one of different sign. We obtain in both cases an antidune, moving towards inflow. The parameters are the following: $\rho=1000 \mathrm{~kg} / \mathrm{m}^{3}, \rho_{s}=2612.9 \mathrm{~kg} / \mathrm{m}^{3}, d_{s}=5.8 \cdot 10^{-3} \mathrm{~m}, n=0.04, \tau_{c r}=0, V=10^{-3} \mathrm{~m} / \mathrm{s}$, $c=8, K_{d}=0.1, K_{e}=1$. The topography is taken as $z_{b}=0.5+0.1 \exp \left(-(x-5)^{2}\right), z_{m}=0.45$, and $z_{f}=z_{b}-z_{m}\left(z_{r}=0\right), \varphi=0, m_{1}=0, m_{2}=3 / 2$ and the flow is initially defined by

$$
\left\{\begin{array}{l}
q=0.4 \\
\frac{u^{2}}{2}+g\left(h+z_{b}\right)=10.13 .
\end{array}\right.
$$

Open boundary conditions have been used. 


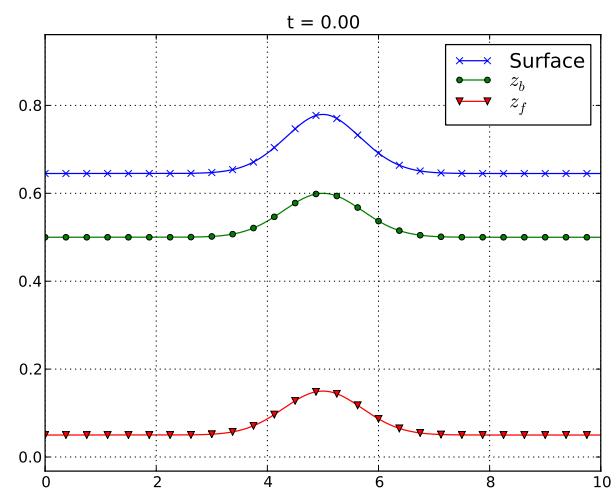

(a) Initial conditions

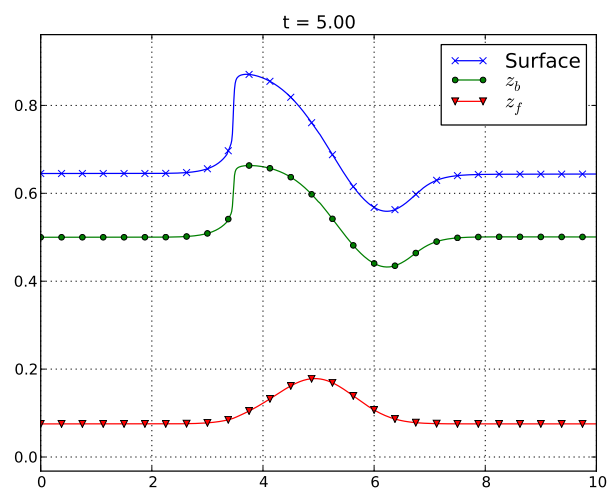

(b) Solution at $t=5$

Figure 9: Free surface, bottom surface $\left(z_{b}\right)$ and layer of sediments that are not moving $\left(z_{f}\right)$ in a supercritical case for which there are two positive and one negative eigenvalues with the new bedload transport discharge

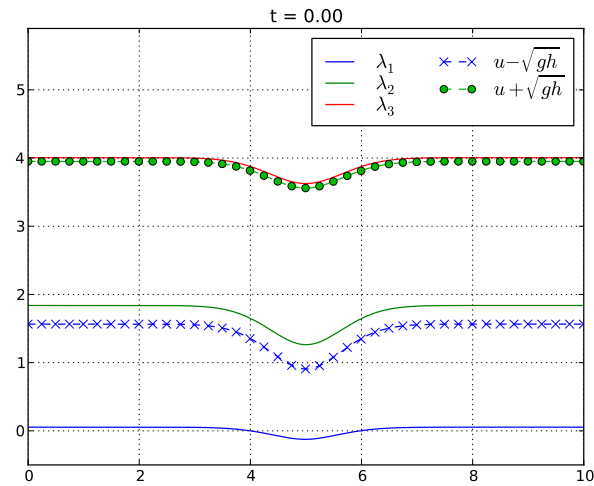

(a) Eigenvalues $t=0$

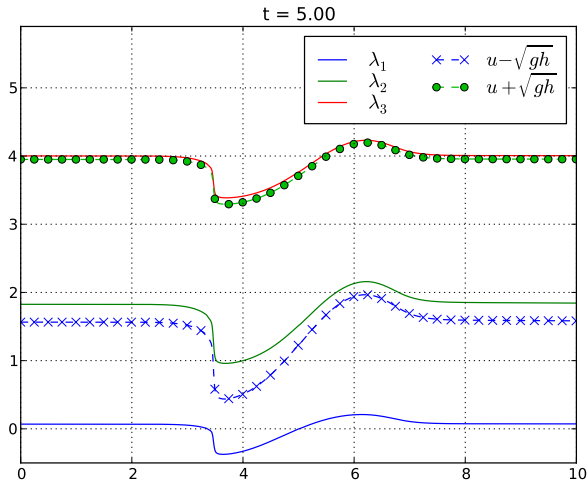

(b) Solution at $t=5$

Figure 10: Comparison between the eigenvalues of System (2) and the one of the Shallow Water system in a supercritical case for which there are two positive and one negative eigenvalues with the new bedload transport discharge

We also run another test where we only change the value of $z_{m}: z_{m}=0.1$.

In this case, we obtain three positive eigenvalues, see Figures 11-12. The bump is going upstream: it is a case of antidune. But this configuration cannot be obtained with a classical formula for the transport discharge.

\subsubsection{Validation thanks to an analytical solution}

In the spirit of [37], we obtained a family of analytical solutions, given by equations (59)-(60). The derivation is detailed in Appendix B. With these solutions, we validate our code for the new bedload formula. We plot in Figure 13 the analytical solution and the approximate solution for $q=0.5$, $A=0.1, B=0.005, C=0.1, K_{d}=1, K_{e}=1, V=5 \cdot 10^{-3} \mathrm{~m} / \mathrm{s}, d_{s}=5.8 \cdot 10^{-3} \mathrm{~m}, \varphi=0, m_{2}=3 / 2$, $C_{1}=100$ and $\left(\frac{u^{2}}{2}+g\left(h+z_{b}\right)\right)_{\mid t=0}=20$ (recall that $m_{1}=0, z_{r}=0$ and $\left.\tau_{c r}^{*}=0\right)$. On the left 


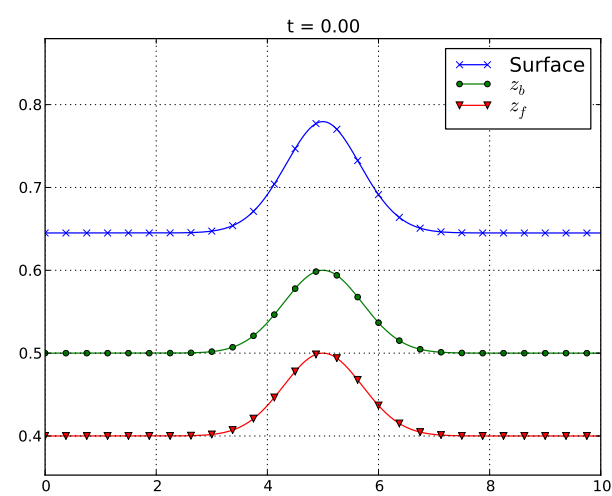

(a) Initial conditions

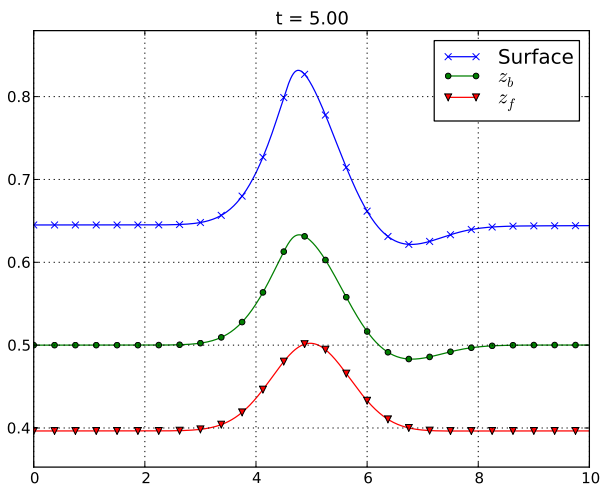

(b) Solution at $t=5$

Figure 11: Free surface, bottom surface $\left(z_{b}\right)$ and layer of sediments that are not moving $\left(z_{f}\right)$ in a supercritical case for which there are three positive eigenvalues with the new bedload transport discharge

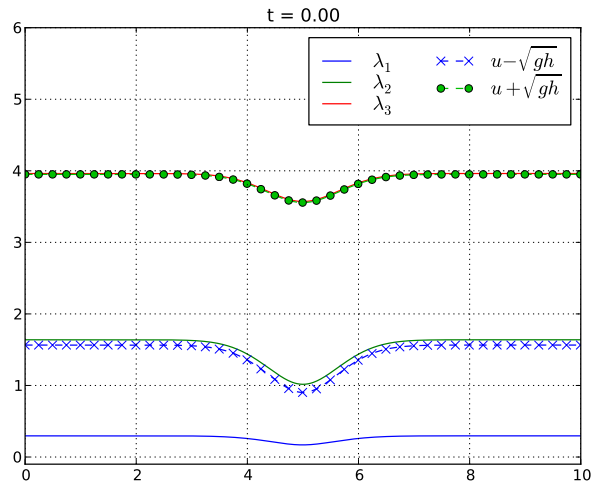

(a) Eigenvalues $t=0$

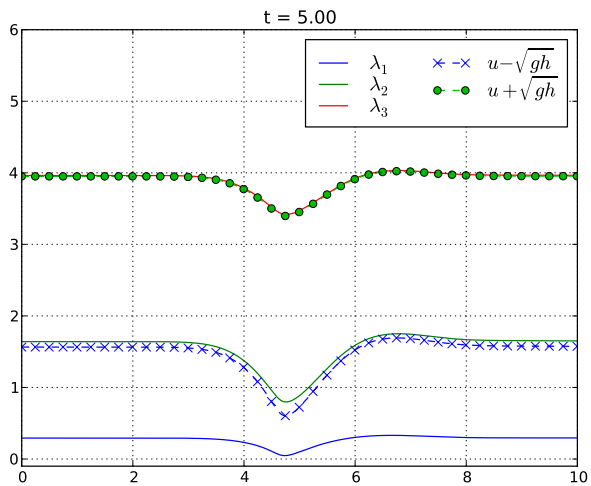

(b) Solution at $t=5$

Figure 12: Comparison between the eigenvalues of System (2) and the one of the Shallow Water system in a supercritical case for which there are three positive eigenvalues with the new bedload transport discharge

boundary, we impose $q=0.5$ and $z_{m}=0.0107$, and on the right boundary the discharge is fixed $q=0.5$.

The two solutions (exact and approximate) coincide.

\subsubsection{Case of a rectangular dune: value of $\tau_{c r}^{*}$}

We carry on the numerical tests with a rectangular dune, as in [9]. When we consider a value $\tau_{c r}^{*}$, we observe that there is a point at the beginning of the dune that distinguish into two different zones: the part of the dune that does not move and the one that is moving. The goal of this test is to study this point, which we may call the rupture point, and its relation with the value of $\tau_{c r}^{*}$.

For this numerical experiment, we consider Darcy-Weisbach friction term with $f=0.1$, the characteristic velocity $V=10^{-2} \mathrm{~m} / \mathrm{s}$; the porosity is $\varphi=0$, and the diameter of the sediments is $d_{s}=5.8 \cdot 10^{-2} \mathrm{~m}$. 


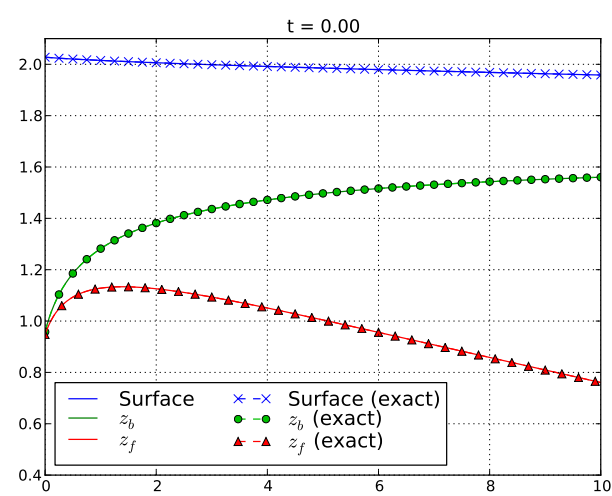

(a) Initial conditions

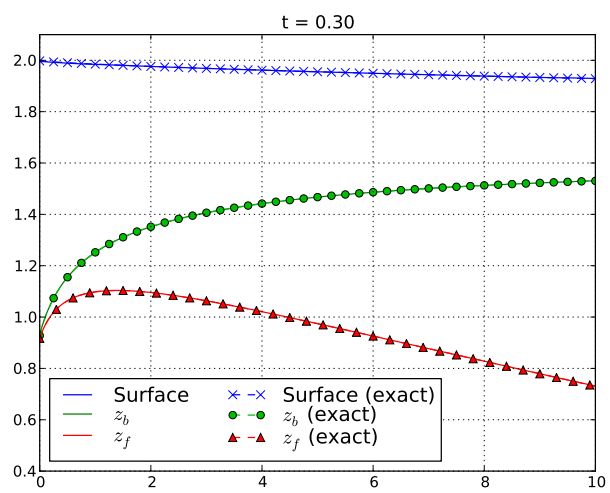

(b) Solution at $t=0.3$

Figure 13: Comparison between the approximate and the analytic solution: free surface, bottom surface $\left(z_{b}\right)$ and layer of sediments that are not moving $\left(z_{f}\right)$

For the bedload transport formula (13), we take $c=8, m_{1}=0$ and $m_{2}=3 / 2$, and, for the erosion and deposition rates, we take $K_{d}=0.1$ and $K_{e}=1$.

The initial conditions are given by the subcritical solution of

$$
\left\{\begin{array}{l}
q=0.2, \\
\frac{u^{2}}{2}+g\left(h+z_{b}\right)=10.42,
\end{array}\right.
$$

with $z_{m}=0, z_{r}=0$ and $z_{b}=0.1+0.5 \cdot \mathbb{1}_{[4,6]}(x)$. We impose $q=0.2$ on the left boundary, and we run several tests with $\tau_{c r}^{*}$ varying from 0.008 to 0.027 , see Figure 14.

At the rupture point, which is located at $x=3$, one expects that $\tau_{b}^{*}=\tau_{c r}^{*}$ for long times and that the Shallow Water relations (56) are satisfied. This is shown in Figure 15. Remark that these relations are equivalent for the classical and new model.

\subsubsection{Case of a dune in one dimension}

The first goal of this test is to study the respective localizations of erosion and deposition. In [38], the authors claims that bumps and upwind slopes get more eroded than dips and downwind faces; that is what we want to check.

We split this test in two parts, depending on the flow regime.

Subcritical case. We choose to run the code over a submerged dune over a flat bottom. More precisely, we take the following parameters: we consider (8) and a Manning formula with coefficient $n=0.04$, the characteristic velocity $V=10^{-3} \mathrm{~m} / \mathrm{s}$; the porosity is $\varphi=0$, the densities are $\rho=1000 \mathrm{~kg} / \mathrm{m}^{3}$ for the water and $\rho_{s}=2612.9 \mathrm{~kg} / \mathrm{m}^{3}$ for the sediments, and the diameter of the sediments is $d_{s}=5.8 \cdot 10^{-5} \mathrm{~m}$. For the bedload transport formula (13), we take $c=8, m_{1}=0$ and $m_{2}=3 / 2$, and, for the erosion and deposition rates, we take $K_{d}=0.1$ and $K_{e}=1$. 


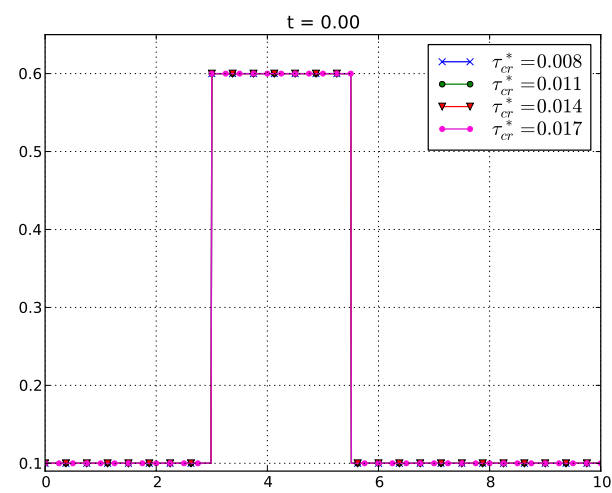

(a) Bottom at $t=0$

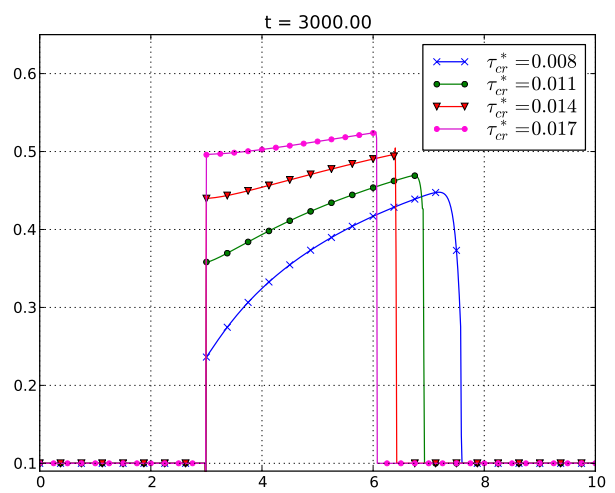

(b) Bottom at $t=3000$

Figure 14: Bottom surface $\left(z_{b}\right)$ with the new bed load transport formula for the evolution of a rectangular dune, with three different values of $\tau_{c r}^{*}$

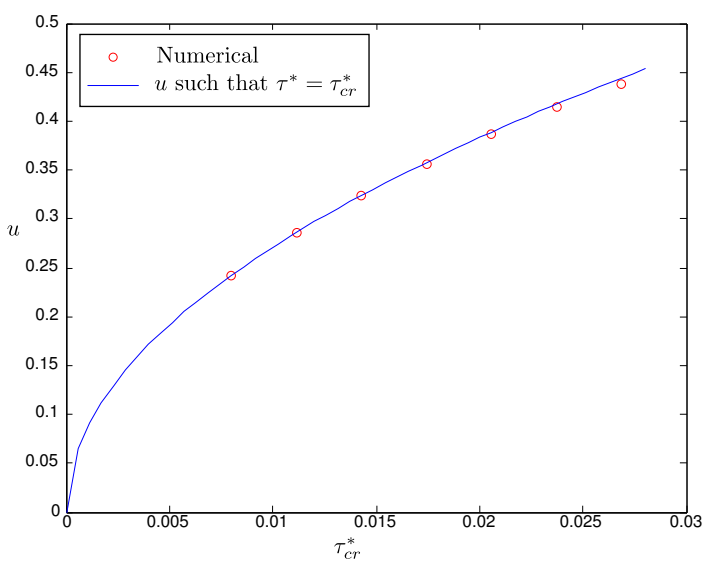

(a) $u$ at $x=3$

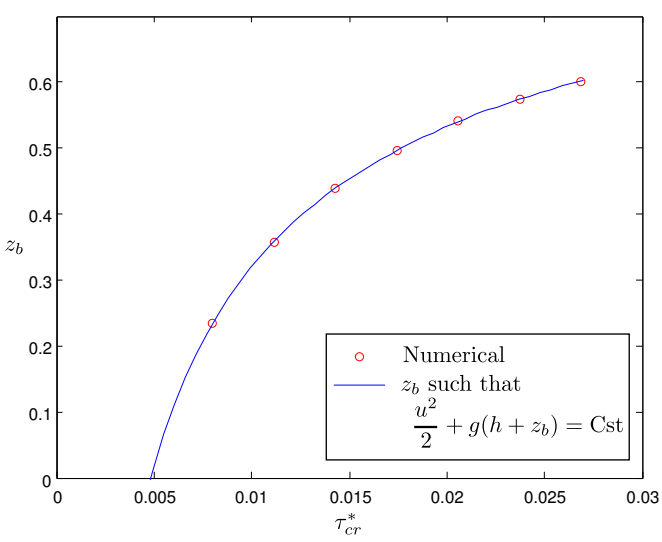

(b) $z_{b}$ at $x=3$

Figure 15: Comparison between theoretical an numerical values at rupture point $x=3$

We start from a stationary state for Shallow Water, namely the subcritical solution of:

$$
\left\{\begin{array}{l}
q=0.5, \\
\frac{u^{2}}{2}+g\left(h+z_{b}\right)=10.42,
\end{array}\right.
$$

and we impose the value of $q=0.5$ on the left boundary (subcritical flow).

The bottom $z_{b}$ is chosen as $z_{b \mid t=0}=0.1+0.1 \exp \left(-(x-5)^{2}\right)$, without non-erodible fixed layer $\left(z_{r}=0\right)$, and we take $z_{m \mid t=0}=\frac{K_{e}}{K_{d}} d_{s}\left(\tau_{b \mid t=0}^{*}-\tau_{c r}^{*}\right)_{+}$in order to even out erosion and deposition at the initial time.

We give in Figure 16 the results at time $t=50$. The difference between erosion and deposition rates is drawn. A positive value means that erosion is predominant, whereas when the value is negative, deposition is predominant. The dashed line represents the values of the bottom $z_{b}$ (values given by 


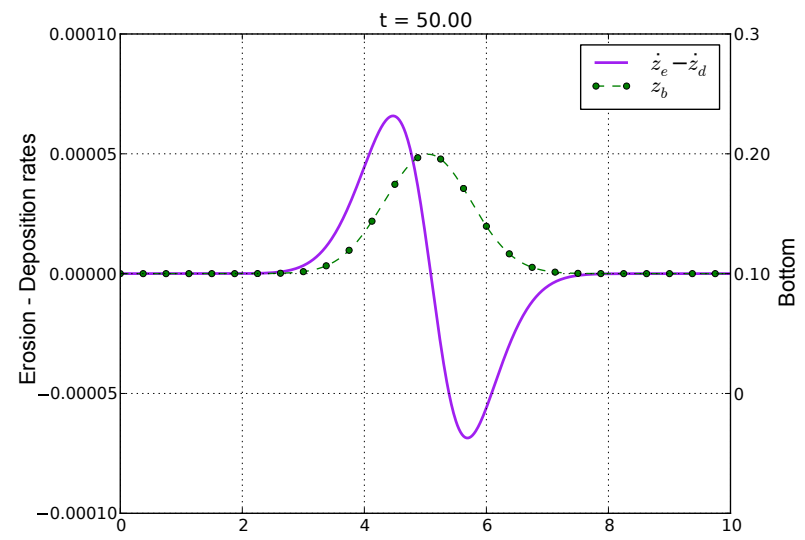

Figure 16: Erosion and deposition rates for a dune (left axis). Bottom is drawn (right axis) to show its influence

the right scale). As said by Andreotti et al., erosion is predominant on the left part of the dune (the flow is accelerated, there is an increase of the shear), and deposition is effective on the right part of the dune.

In that case, we can compare the behavior of the new and the classical solid transport discharges. Indeed, this one-dimensional dune is a test case that returns non-physical results for a lot of numerical models, giving a straight front of the dune. The usual way to get acceptable results is to change the numerical resolution by adding some numerical viscosity.

We present in Figure 17 the results at times 300, 1000, 2000, 4000 with the two formulations for the solid discharge.

We notice that the new formulation is more realistic than the classical one. On the right part of the dune indeed, the straight line given by the classical model is not physical, which is corrected by the deposition in the proposed model.

We can also plot the boundary between the layer of moving particles and the layer of sediments that are not moving, see Figure 18.

Supercritical case. We turn out to study this dune case for a supercritical regime, choosing the characteristic velocity $V=10^{-5} \mathrm{~m} / \mathrm{s}$, the diameter of the sediments is $d_{s}=10^{-4} \mathrm{~m}$ and for the erosion and deposition rates, we take $K_{d}=0.04$ and $K_{e}=0.1$ (other parameters remain unchanged).

We start from a supercritical stationary state for Shallow Water, solution of:

$$
\left\{\begin{array}{l}
q=1.25, \\
\frac{u^{2}}{2}+g\left(h+z_{b}\right)=10.42,
\end{array}\right.
$$

and we impose the value of $q=1.25$ on the left boundary. 


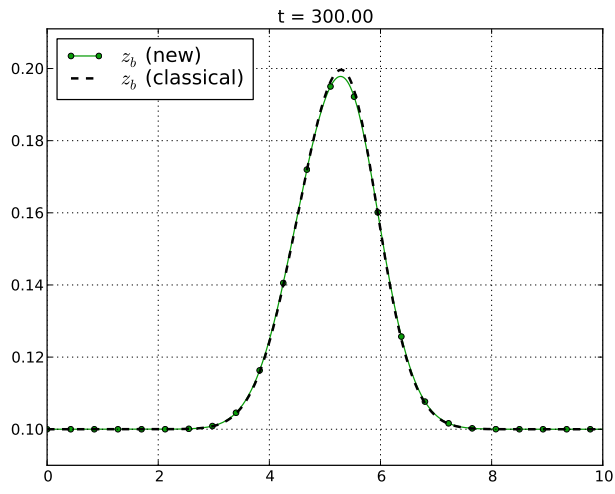

(a) Comparison at $t=300$

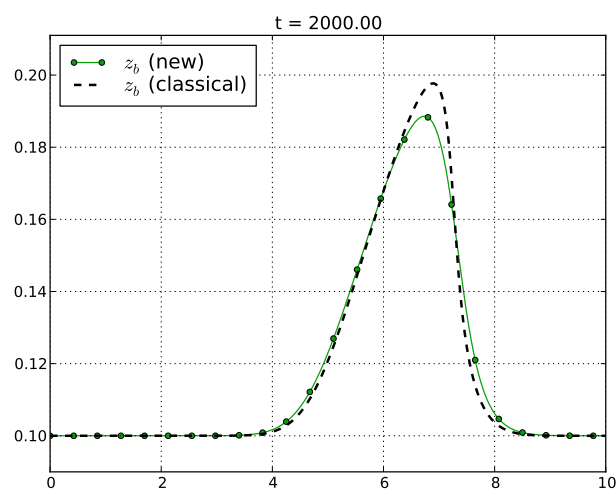

(c) Comparison at $t=2000$

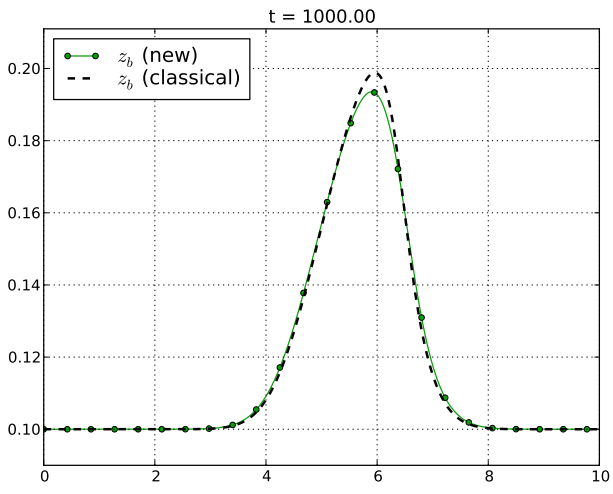

(b) Comparison at $t=1000$

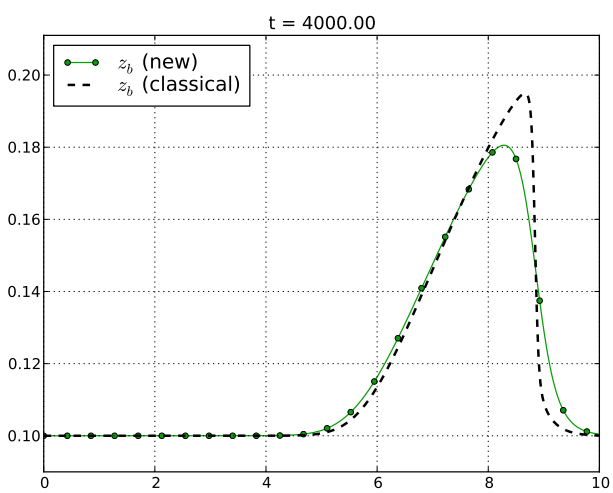

(d) Comparison at $t=4000$

Figure 17: Bottom surface $\left(z_{b}\right)$ with the new and classical bedload transport formulae for the evolution of a dune 


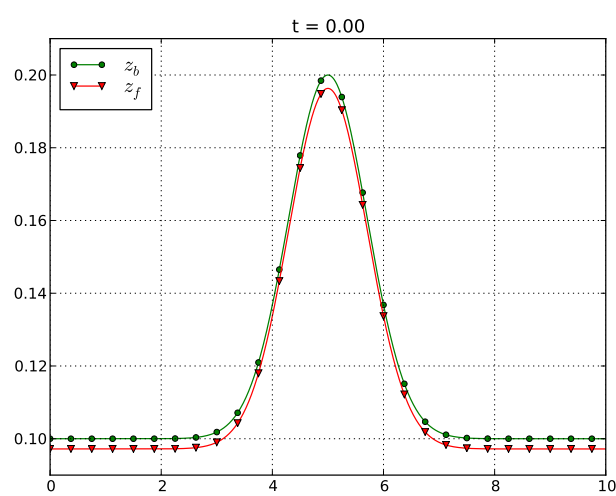

(a) Bottom at $t=0$

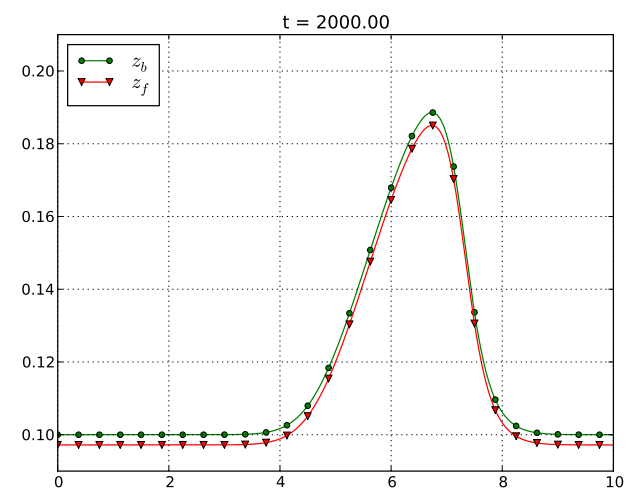

(b) Bottom at $t=2000$

Figure 18: Bottom surface $\left(z_{b}\right)$ and layer of sediments that are not moving $\left(z_{f}\right)$ with the new bedload transport formula for the evolution of a dune

Results are given in Figures 19-20: in Figure 19, we plotted the two bottom layers, the water height and the difference between erosion and deposition rates for various values of the time. In Figure 20, we complete the previous results with the square of the Froude number, giving a visual criterion to known the flow regime (whether it is greater than one or not).

For small time values, the deposition is greater than the erosion at the top of the dune and reversed far from the top of the dune. As a consequence the thickness of the sediment layer $z_{m}$ increases faster at the top of the dune which generates a hydraulic jump which propagates upstream. As the shock leaves the domain, the fluid changes from subcritical (upstream of the dune) to supercritical (downstream). Erosion is then grater than deposition downstream of the dune while it is reversed upstream. The assertion given by Andreotti et al. in not valid in this supercritical case anymore: deposition is now predominant on the left part of the dune (upwind slope).

\subsubsection{Case of a rectangular dune in two dimensions}

We carry on the numerical tests with a rectangular dune, in two dimensions. For this numerical experiment, we consider the rectangular domain $[1,10] \times[-0.5,0.5]$ with 80 finite volumes in the $x$-direction and 50 finite volumes in the $y$-direction. A second order scheme has been used for the simulations. We have performed this test for a non-trivial $z_{r}$, given by $z_{r}=0.5-0.01 x$, with $z_{f}=0.15 \cdot \mathbb{1}_{[3,5]}(x) \cdot \mathbb{1}_{[-0.2,0.2]}(y), z_{m}=0.05 \cdot \mathbb{1}_{[3,5]}(x) \cdot \mathbb{1}_{[-0.2,0.2]}(y)$. The values of the parameters are: $d_{s}=5.8 \cdot 10^{-4} \mathrm{~m}, V=10^{-3} \mathrm{~m} / \mathrm{s}, K_{d}=0.5$ and $K_{e}=5, c=40, m_{1}=0, m_{2}=3 / 2$ and $\tau_{c r}^{*}=0.5$. We choose a Manning friction term with $n=0.04$. On the left boundary, we impose $q=0.25$ and $z_{m}=0$, on the right boundary, we impose $h=0.6101$, and the initial solution corresponds to a subcritical 


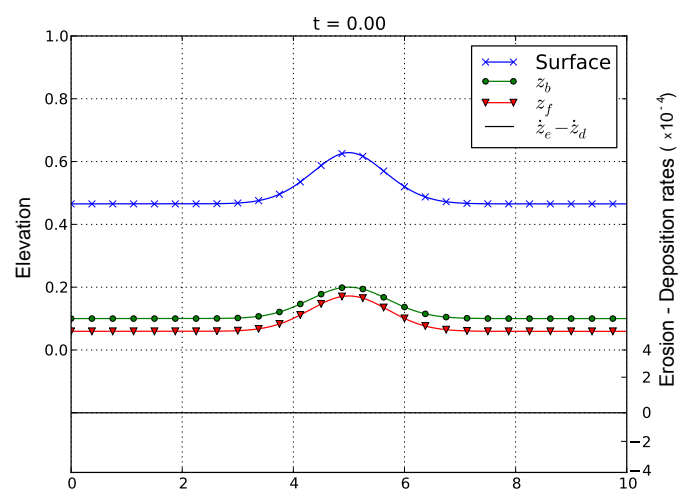

(a) initial condition

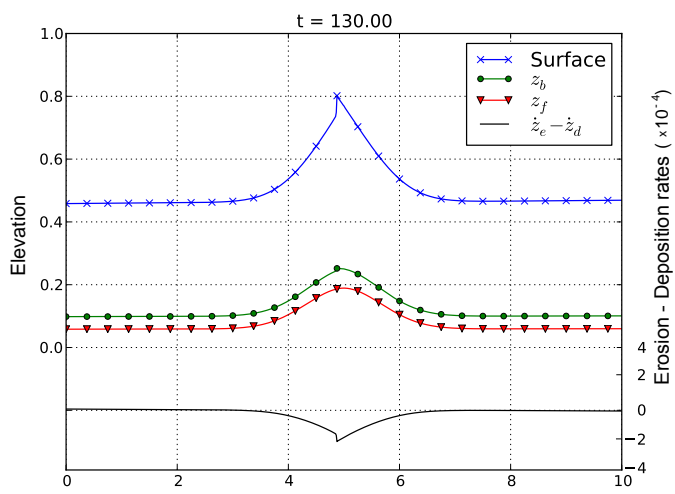

(c) Evolution at $t=130$

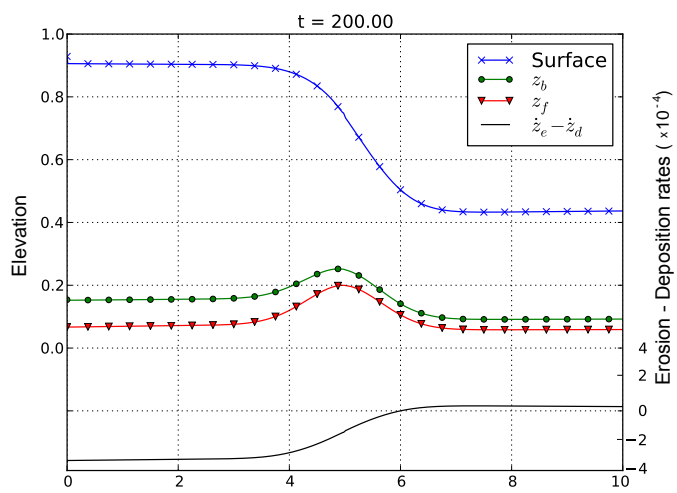

(e) Evolution at $t=200$

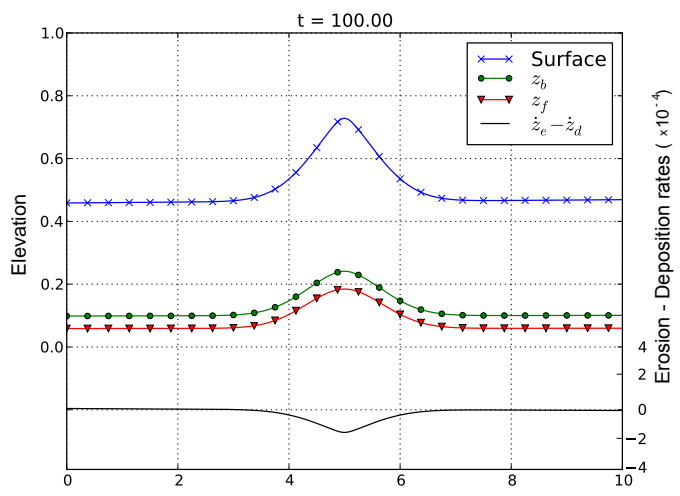

(b) Evolution at $t=100$

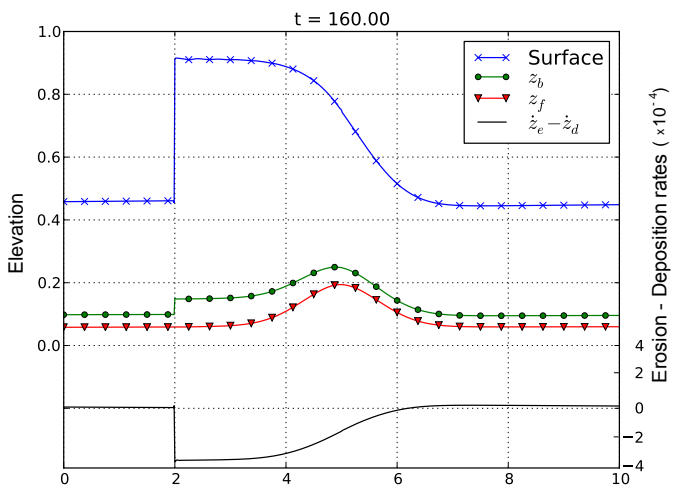

(d) Evolution at $t=160$

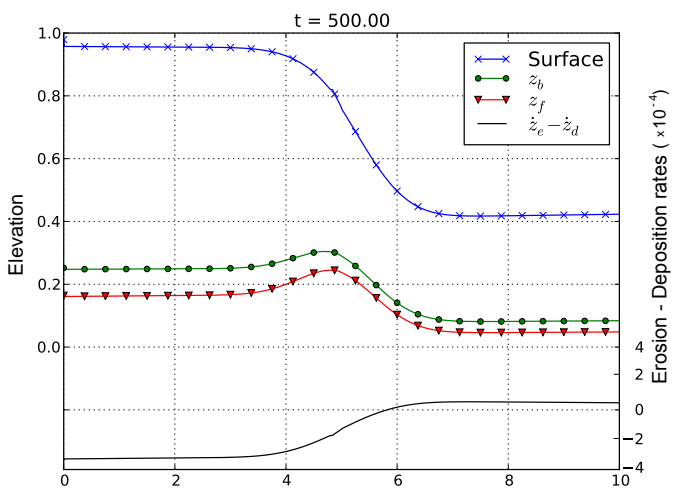

(f) Evolution at $t=500$

Figure 19: Evolution of a dune in an initial supercritical regime. The left axis represents the height for surface and bottom and the right axis represents the difference between erosion and deposition rates 


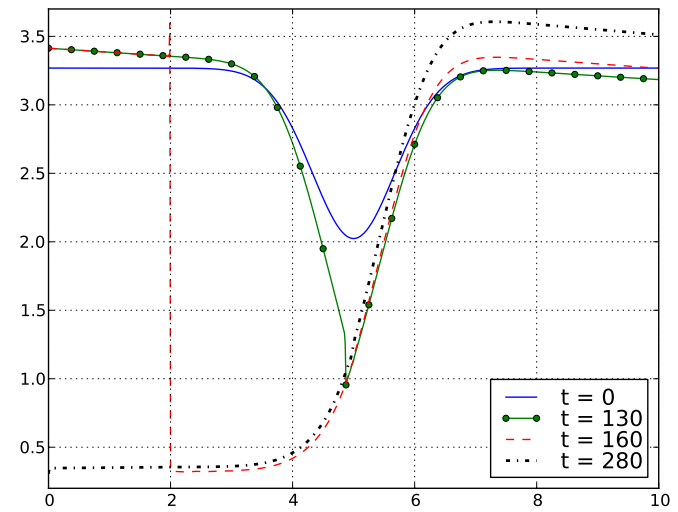

Figure 20: Square of the Froude number (i.e. $u^{2} /(g h)$ ) for the dune in an initial supercritical regime, at several times. When it is greater than 1 , the flow is supercritial, whereas on the contrary, the flow is subcritical

regime satisfying

$$
\left\{\begin{array}{l}
q=0.25 \\
\frac{u^{2}}{2}+g\left(h+z_{b}\right)=10 .
\end{array}\right.
$$

The results are given in Figure 21, and we can see erosion effects on the left part of the dune, deposition effects on the right part. More precisely, this test shows several results. First, starting from a quite large moving layer $\left(z_{m}=0.05 \mathrm{~m}\right.$ while the dune is $0.2 \mathrm{~m}$ high), the moving layer becomes smaller to attain a size of the order of the diameter of grains. Concerning the profile of the dune, the front was sharp at the initial time but, thanks to deposition, the layer of sediments that are not moving $\left(z_{f}\right)$ increases and it creates a smooth profile.

\subsubsection{Propagation of a sediment bore}

We intend now to apply the new model presented here to a experiment shown in [32] and [39]. The experiment consists in a steep-sloped, rectangular channel of finite length. The bed profile is in quasiequilibrium and a constant sediment supply is fed upstream. At reference time $t=0$, this equilibrium situation is perturbed by the rapid raise of a submerged weir at the downstream end of the flume, imposing a subcritical condition. The water and sediment discharges at the upstream section are kept constant. This hydraulic configuration gives rise to a hydraulic jump and a sediment bore. The flume is $6.9 \mathrm{~m}$ long, $0.50 \mathrm{~m}$ wide and the slope is equal to $3.02 \%$. The sediment and water discharges are $q_{b}=0.136 \mathrm{~L} / \mathrm{s}$ and $q=12 \mathrm{~L} / \mathrm{s}$, respectively. Uniform coarse sand with a mean diameter of $1.65 \mathrm{~mm}$ and with a porosity of 0.42 is considered. Finally, Manning coefficient is $n=0.0165 \mathrm{~m}^{-1 / 3} \mathrm{~s}$. We refer to [32] and [39] for a detailed description of the experiments. We remark that we lack some of the information needed when applying the new solid transport discharge introduced here as we do not know information such as the thickness of the sediment layer $z_{m}$ or the parameters that define the 


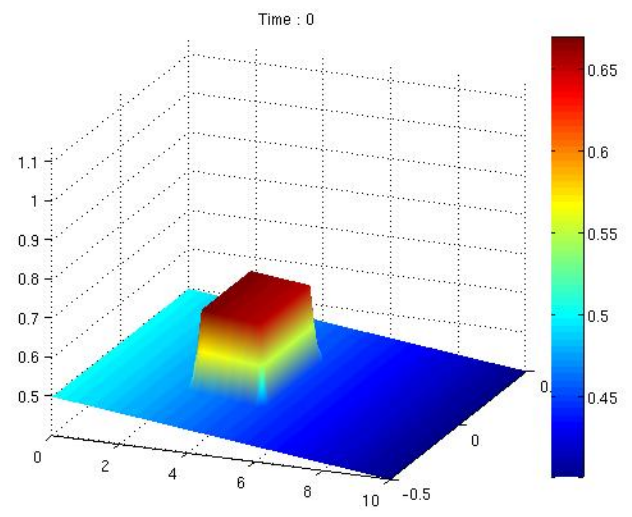

(a) Initial topography

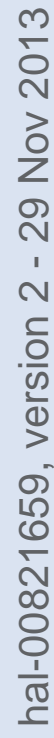

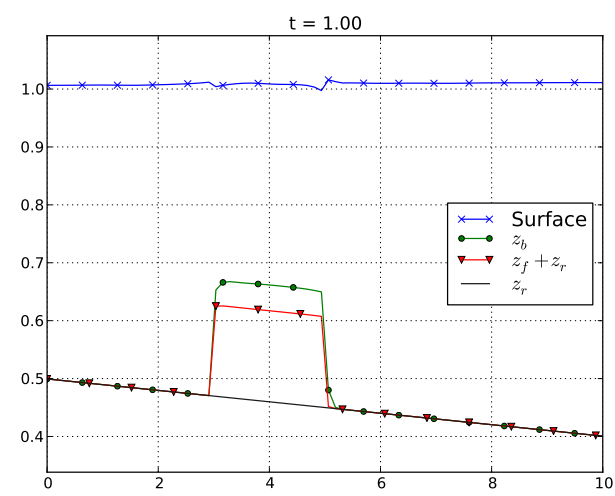

(c) Solution for $y=0$ at $t=1$

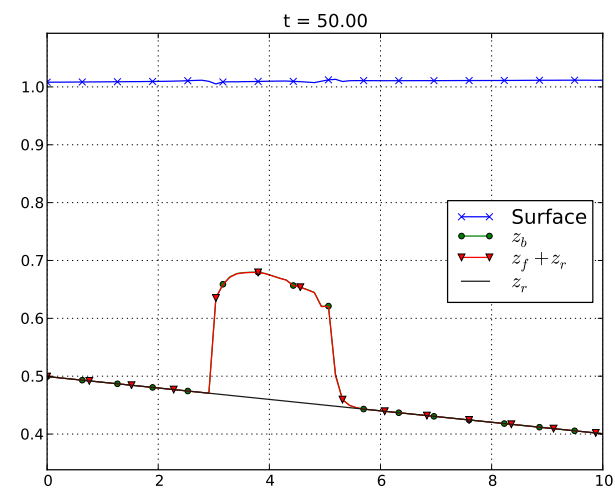

(e) Solution for $y=0$ at $t=50$

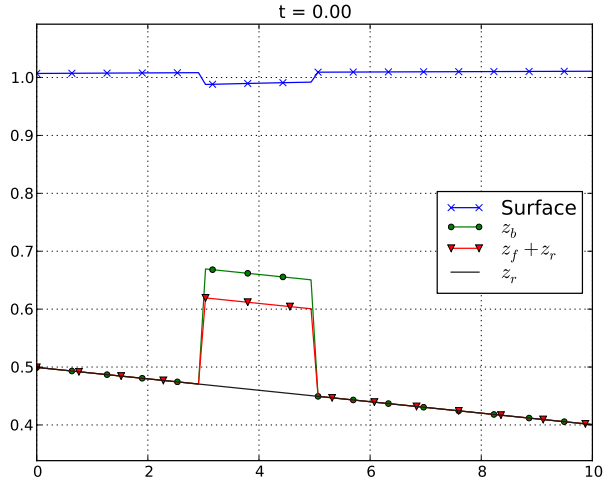

(b) Initial conditions for $y=0$

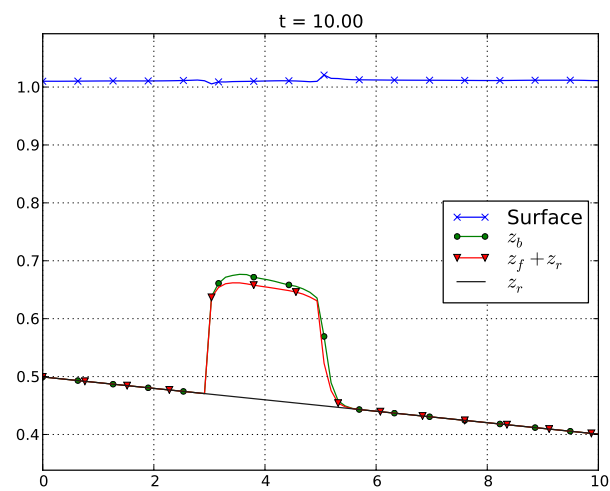

(d) Solution for $y=0$ at $t=10$

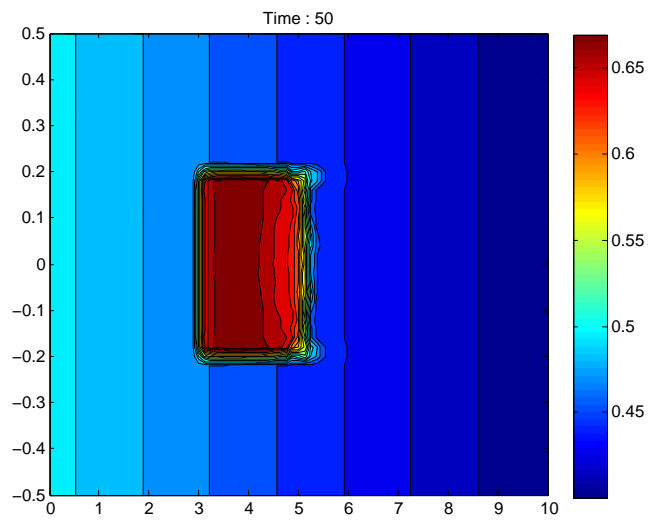

(f) Solution at $t=50$

Figure 21: Free surface, bottom surface $\left(z_{b}\right)$, layer of sediments that are not moving $\left(z_{f}\right)$ and bedrock layer $\left(z_{r}\right)$ with the new bedload transport formula for the evolution of a two dimensional rectangular dune on an inclined plane 


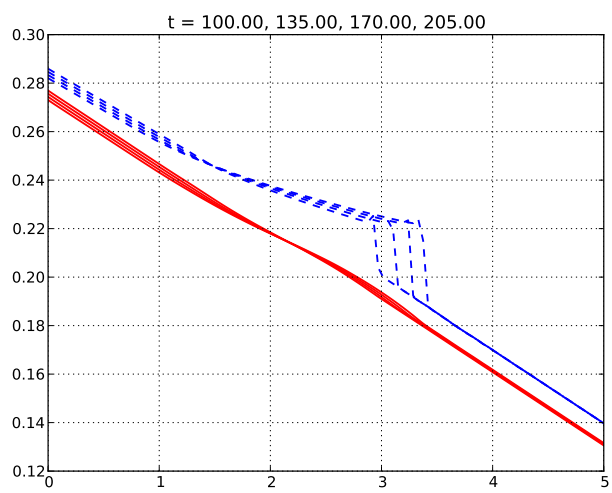

(a) Evolution of the bed topography (zoom)

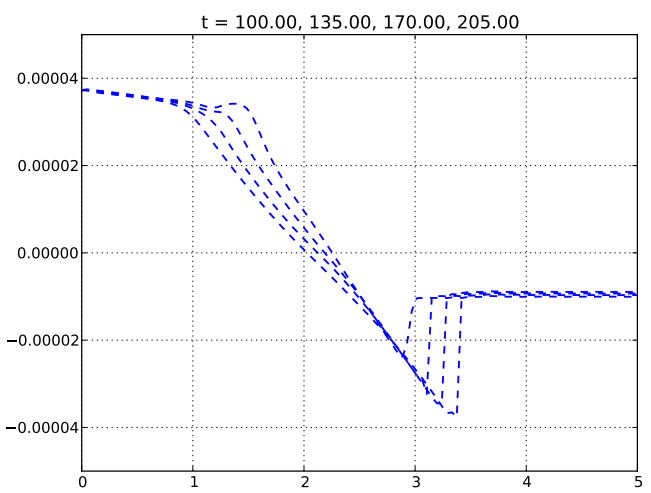

(b) Erosion-Deposition rates

Figure 22: Position of the bore (left) every $35 \mathrm{~s}$ from $t=100$ to $t=205$ and erosion-deposition rates (right). In left picture, dashed and continuous lines represent the bottom surface $\left(z_{b}\right)$ and the layer of sediments that are not moving $\left(z_{f}\right)$ respectively. In right picture, positive values correspond to regions where erosion is predominant over deposition

erosion (16) and deposition (14) rates. For practical purposes we set an initial thickness $z_{m}=0.01$. We then run the test for different ratios between erosion and deposition rates. Then, the position of the bore is obtained as in [32] and compared to experimental data. Evolution of the bore and erosiondeposition rates are shown in Figure 22 and the front position is shown in Figure 23 for different ratios of erosion and deposition (18). In general, we see that in spite of uncertainty of some parameters, the numerical results agree with experimental data. Nevertheless, we remark that the speed of the bore is reduced for long times. This is due to the fact that deposition is stronger than erosion downstream, which means that $z_{m}$ is progressively reduced which means that the velocity of the sediment layer is reduced. We conclude thus that a correct parametrization of the erosion and deposition rates is crucial as the speed of the bore strongly depends on them.

\section{Conclusions}

We have introduced a general formulation for solid transport flux that takes into account the thickness of the sediment layer. Compared to classical formulae this one has the advantage of preserving sediment mass in situations where sediment is isolated inside the considered domain. This new formulation reduces to the classical one when we consider quasi-uniform regimes. Moreover, it considers two layers of sediment: one that is actually moving due to the fluid and one that is not moving but could be entrained into the moving layer. As a consequence, the ideas taken into account are closer to the physics of the problem. Numerical simulations show that this generalization of solid transport flux is very promising. In particular, we remark that the vertical profiles on the front of an advancing dune (characteristic when using a classical formulation) are avoided and smoothed in a more realistic way. 


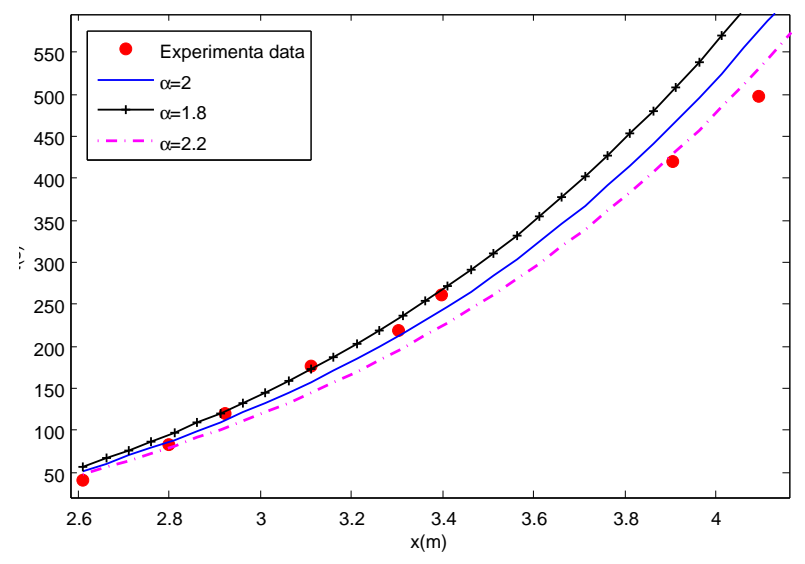

Figure 23: Comparison of front position for different values of $\alpha$ (18)

\section{Appendix A}

\section{Proof of Proposition 4.2}

We define the two tangents of the curve $f(\lambda)$ which are parallel to $d(\lambda)$. Their intersections with $f(\lambda)$ are characterized by $f^{\prime}(\lambda)=g h b$ which yields to two values of $\lambda$ of the form

$$
\lambda_{ \pm} \stackrel{\text { def }}{=} \frac{2 u+v_{b} \pm \sqrt{\left(u-v_{b}\right)^{2}+3(g h+g h b)}}{3} .
$$

The two tangents are such that $d_{ \pm}\left(\lambda_{ \pm}\right)=f\left(\lambda_{ \pm}\right)$. This implies the equations for the tangents are given by

$$
d_{ \pm}(\lambda)=g h b\left(\lambda-\alpha_{ \pm}\right)
$$

with

$$
\alpha_{ \pm} \stackrel{\text { def }}{=} \lambda_{ \pm}-\frac{f\left(\lambda_{ \pm}\right)}{g h b}
$$

The roots of $p_{A}(\lambda)$ which correspond to the eigenvalues of $A(W)$ are given as the intersection of $f(\lambda)$ and $d(\lambda)$ (see Figure 4).

Recall that $f(\lambda)$ is a third order polynomial with roots $\left\{\mu_{1}, \mu_{2}, \mu_{3}\right\}=\left\{v_{b}, u \pm \sqrt{g h}\right\}$. The equation $p_{A}(\lambda)=d(\lambda)-f(\lambda)$ will have 3 distinct solutions if and only if the line $d(\lambda)$ lies in between $d_{-}(\lambda)$ and $d_{+}(\lambda)$. This can be equivalently written as $\alpha_{-}<k u<\alpha_{+}$.

It can be checked that we always have $\alpha_{-}<u-\sqrt{g h}<u<u+\sqrt{g h}<\alpha_{+}$so the system is always hyperbolic in the case $k=1$. In the case $k=7 / 6$, if $|u|<6 \sqrt{g h}$, we have $u-\sqrt{g h}<\frac{7}{6} u<u+\sqrt{g h}$ and thus the hyperbolicity condition is verified which concludes the proof. 


\section{Appendix B}

\section{Analytical solutions}

Following [37], we can obtain analytical solutions for System (2). Considering the new solid transport discharge, a constant water discharge $q=h u$ and a water height that only depends on $x(i . e . h=h(x)$ ), then $u=u(x)=q / h(x)$, System (2) reduces to:

$$
\left\{\begin{array}{l}
q=c s t \\
\partial_{t} h=0 \\
\partial_{x}\left(\frac{u^{2}}{2}+g\left(h+z_{b}\right)\right)=0 \\
\partial_{t} z_{b}+\partial_{x} \widetilde{q}_{b}=0 \\
\partial_{t} z_{f}=\dot{z}_{d}-\dot{z}_{e}
\end{array}\right.
$$

Differentiating the third equation of System (58) with respect to time, we get: $\partial_{t x} z_{b}=0$. Then, differentiating the fourth equation with respect to space, we obtain: $\partial_{x x} \widetilde{q}_{b}=0$, and

$$
\widetilde{q}_{b}=A(t) x+B(t)=z_{m} \alpha c Q \operatorname{sgn}\left(\tau_{b}\right)\left(\tau_{b}^{*}\right)^{m_{1}}\left(\tau_{b}^{*}-\tau_{c r}^{*}\right)_{+}^{m_{2}-1}=z_{m} C\left(\tau_{b}^{*}\right)^{m_{1}}\left(\tau_{b}^{*}-\tau_{c r}^{*}\right)_{+}^{m_{2}-1}
$$

In order to simplify the computations, we use Darcy-Weisbach friction law (in this case, $S_{\mathrm{f}}$ is a function of $u$ only and does not depend on $h$ ) and we rewrite $\tau_{b}^{*}$ as :

$$
\tau_{b}^{*}=\frac{f u^{2}}{8\left(\frac{\rho_{s}}{\rho}-1\right) g d_{s}}=C_{1} u^{2} .
$$

Let us assume that $m_{1}=0, \tau_{c r}^{*}=0$; then

$$
\widetilde{q}_{b}=A(t) x+B(t)=z_{m} C\left(C_{1} u^{2}\right)^{m_{2}-1} .
$$

As $\left(C_{1} u^{2}\right)^{m_{2}-1}$ does not depend on the time, we have:

$$
0=\partial_{t} \frac{A(t) x+B(t)}{z_{m}}=\frac{\left(A^{\prime}(t) x+B^{\prime}(t)\right) z_{m}+(A(t) x+B(t)) \partial_{t} z_{m}}{z_{m}^{2}},
$$

and

$$
\partial_{t} z_{m}=\frac{A^{\prime}(t) x+B^{\prime}(t)}{A(t) x+B(t)} z_{m}
$$

The last equation of System (58) reads:

$$
\partial_{t} z_{f}=-\dot{z}_{e}+\dot{z}_{d}=-K_{e} \frac{V}{1-\varphi}\left(\tau_{b}^{*}-\tau_{c r}^{*}\right)_{+}+K_{d} V \frac{z_{m}}{d_{s}}=-K_{e} \frac{V}{1-\varphi} C_{1} u^{2}+K_{d} V \frac{z_{m}}{d_{s}}
$$

then

$$
-A(t)=-\partial_{x} \widetilde{q}_{b}=\partial_{t} z_{b}=\partial_{t}\left(z_{f}+z_{m}\right)=-K_{e} \frac{V}{1-\varphi} C_{1} u^{2}+K_{d} V \frac{z_{m}}{d_{s}}+\frac{A^{\prime}(t) x+B^{\prime}(t)}{A(t) x+B(t)} z_{m} .
$$


If we choose $A(t)=A$ and $B(t)=B$ two constants, then $z_{m}$ does not depend on time and we obtain:

$$
\left\{\begin{array}{l}
-A=-K_{e} \frac{V}{1-\varphi} C_{1} u^{2}+K_{d} V \frac{z_{m}}{d_{s}} \\
A x+B=z_{m} C\left(C_{1} u^{2}\right)^{m_{2}-1}
\end{array}\right.
$$

that is

$$
\left\{\begin{array} { l } 
{ K _ { e } \frac { V } { 1 - \varphi } C _ { 1 } u ^ { 2 } = A + K _ { d } V \frac { z _ { m } } { d _ { s } } , } \\
{ A x + B = z _ { m } C ( C _ { 1 } u ^ { 2 } ) ^ { m _ { 2 } - 1 } , }
\end{array} \text { or } \left\{\begin{array}{l}
C_{1} u^{2}=\left(A+K_{d} V \frac{z_{m}}{d_{s}}\right) \frac{1-\varphi}{V K_{e}}, \\
A x+B=z_{m} C\left(\left(A+K_{d} V \frac{z_{m}}{d_{s}}\right) \frac{1-\varphi}{V K_{e}}\right)^{m_{2}-1} .
\end{array}\right.\right.
$$

Let us summarize the formulae: at the initial time,

$$
\left\{\begin{array}{l}
q=c s t \\
z_{m} \text { is the solution of } A x+B=z_{m} C\left(\left(A+K_{d} V \frac{z_{m}}{d_{s}}\right) \frac{1-\varphi}{V K_{e}}\right)^{m_{2}-1} \\
u=\sqrt{\left(A+K_{d} V \frac{z_{m}}{d_{s}}\right) \frac{1-\varphi}{V K_{e}} \frac{1}{C_{1}}} \\
h=\frac{q}{u} \\
\partial_{t} z_{b}=-A \text { with } z_{b \mid t=0} \text { given by }\left(\frac{u^{2}}{2}+g\left(h+z_{b}\right)\right)_{\mid t=0}=c s t
\end{array}\right.
$$

and the evolution in time is performed as

$$
z_{f}=-A t+z_{f \mid t=0}=-A t+z_{b \mid t=0}-z_{m}
$$

\section{Acknowledgments}

This research has been partially supported by the Spanish Government and FEDER through the Research project MTM2012-38383-C02-02, by the Andalusian Government through the project P11RNM7069 and by the French CNRS via GdR EGRIN. E.D. F-N would likes to thank the MAPMO for the invitation of one month in the University of Orléans. The authors would like to thank PierreYves Lagrée for the fruitful discussions. The numerical computations have been performed at the Laboratory of Numerical Methods of the University of Málaga.

\section{References}

[1] D. Simons, F. Şentürk, Sediment Transport Technology: Water and Sediment Dynamics, Water Resources Publ., ISBN 9780918334664, URL http://books.google.fr/books?id=_eScptWZAeIC, 1992.

[2] K. Wilson, Bed-load transport at high shear stress, Journal of the Hydraulics Division 92 (11) (1966) 49-59. 
[3] J. Fredsøe, R. Deigaard, Mechanics of Coastal Sediment Transport, World Scientific Publishing Company, ISBN 9810208413, 1992.

[4] F. Charru, H. Mouilleron, O. Eiff, Erosion and deposition of particles on a bed sheared by a viscous flow, Journal of Fluid Mechanics 519 (2004) 55-80, doi:10.1017/S0022112004001028, URL http://dx.doi.org/10.1017/S0022112004001028.

[5] F. Charru, Selection of the ripple length on a granular bed sheared by a liquid flow, Physics of Fluids 18 (12) 121508, doi:10.1063/1.2397005, URL http://link. aip.org/link/?PHF/18/121508/1.

[6] S. Cordier, C. Lucas, J. d. D. Zabsonré, A two time-scale model for tidal bed-load transport, Communications in Mathematical Sciences 10 (3) (2012) 875-888.

[7] K. Z. Kuai, C. W. Tsai, Identification of varying time scales in sediment transport using the Hilbert-Huang Transform method, Journal of Hydrology 420-421 (2012) 245-254, ISSN 0022-1694, doi:10.1016/j.jhydrol.2011.12.007, URL http://www.sciencedirect.com/science/article/pii/S0022169411008808.

[8] F. Exner, Über die wechselwirkung zwischen wasser und geschiebe in flüssen, Sitzungsber., Akad. Wissenschaften pt. IIa (1925) Bd. 134.

[9] M. Castro Díaz, E. Fernández-Nieto, A. Ferreiro, Sediment transport models in Shallow Water equations and numerical approach by high order finite volume methods, Computers \& Fluids 37 (3) (2008) 299-316, ISSN 0045-7930, doi:10.1016/j.compfluid.2007.07.017, URL http://www.sciencedirect. com/science/article/B6V26-4PSK8R5-1/2/9043410cc90abc43b410c70660a4efd6.

[10] A. M. Ferreiro, Development of post-process technics of hydrodynamics flux, modelization of sediment transport problems and numerical simulation through finite volume technics, Ph.D. thesis, University of Sevilla, 2006.

[11] C. Savary, Transcritical transient flow over mobile beds, boundary conditions treatment in a two-layer shallow-water model, Ph.D. thesis, Louvain, 2007.

[12] B. Toumbou, D. Le Roux, A. Sene, An existence theorem for a 2-D Coupled Sedimentation Shallow-Water Model, C. R. Math. Acad. Sci. Paris 344 (7) (2007) 443-446.

[13] A. Grass, Sediment transport by waves and currents, SERC London Cent. Mar. Technol Report No. FL29.

[14] E. Meyer-Peter, R. Müller, Formulas for bed-load transport, in: 2nd meeting IAHSR, Stockholm, Sweden, 1-26, 1948. 
[15] L. Van Rijn, Sediment transport Part 1: bed load transport., Journal of Hydraulic Engineering - ASCE 110 (10) (1984) 1431-1456.

[16] P. Nielsen, Coastal Bottom Boundary Layers and Sediment Transport, World Scientific Pub Co Inc, ISBN 9810204736, 1992.

[17] A. C. Fowler, N. Kopteva, C. Oakley, The Formation of River Channels, SIAM Journal on Applied Mathematics 67 (4) (2007) 1016-1040, URL http://epubs.siam.org/doi/abs/10.1137/050629264.

[18] T. Morales de Luna, M. J. Castro Díaz, C. Parés Madroñal, A Duality Method for Sediment Transport Based on a Modified Meyer-Peter \& Müller Model, Journal of Scientific Computing 48 (1-3) (2011) 258-273, ISSN 0885-7474, doi:10.1007/s10915-010-9447-1, URL http://www. springerlink. com/content/7401364w29464259/.

[19] A. Shields, Anwendung der Ähnlichkeitsmechanik und der Turbulenzforschung auf die Geschiebebewegung, Tech. Rep. 26, Mitteilungen der Preussischen Versuchsanstalt für Wasserbau und Schiffbau, 1936.

[20] R. Fernandez-Luque, R. van Beek, Erosion and transport of bedload sediment, J. Hydraul. Res. 14 (2) (1976) 127-144.

[21] K. K. J. Kouakou, P.-Y. Lagrée, Evolution of a model dune in a shear flow, European Journal of Mechanics - B/Fluids $25 \quad$ (3) (2006) 348-359, ISSN 0997-7546, doi:10.1016/j.euromechflu.2005.09.002, URL http://www.sciencedirect.com/science/article/pii/S0997754605000737.

[22] H. A. Einstein, R. B. Krone, Experiments to determine modes of cohesive sediment transport in salt water, J. Geophys. Res. 67 (4) (1962) 1451-1461.

[23] R. Bagnold, The Flow of Cohesionless Grains in Fluids, Royal Society of London Philosophical transactions. Series A. Mathematical and physical sciences, no. 964, Royal Society of London, URL http://books.google.fr/books?id=VaqNGwAACAAJ, 1956.

[24] E. Godlewski, P.-A. Raviart, Numerical approximation of hyperbolic systems of conservation laws, vol. 118 of Applied Mathematical Sciences, Springer-Verlag, New York, ISBN 0-387-94529-6, 1996.

[25] S. Cordier, M. Le, T. Morales de Luna, Bedload transport in shallow water models: Why splitting (may) fail, how hyperbolicity (can) help, Advances in Water Resources 34 (8) (2011) 980-989, ISSN 0309-1708, doi:16/j.advwatres.2011.05.002, URL http://www.sciencedirect.com/science/article/pii/S0309170811000935. 
[26] T. Morales de Luna, M. J. Castro Díaz, C. Parés Madroñal, E. D. Fernández Nieto, On a shallow water model for the simulation of turbidity currents, Communications in Computational Physics 6 (4) (2009) 848-882, doi:10.4208/cicp.2009.v6.p848, URL http://www.global-sci.com/cgi-bin/fulltext/6/848/full.

[27] C. Parés, Numerical methods for nonconservative hyperbolic systems: a theoretical framework, SIAM J. Numer. Anal. 44 (1) (2006) 300-321 (electronic), ISSN 0036-1429.

[28] I. Toumi, A weak formulation of Roe's approximate Riemann solver, J. Comput. Phys. 102 (2) (1992) 360-373.

[29] M. Castro, J. Macías, C. Parés, A Q-scheme for a class of systems of coupled conservation laws with source term. Application to a two-layer 1-D shallow water system, M2AN Math. Model. Numer. Anal. 35 (1) (2001) 107-127.

[30] C. Parés, M. Castro, On the well-balance property of Roe's method for nonconservative hyperbolic systems. Applications to shallow-water systems, M2AN Math. Model. Numer. Anal. 38 (5) (2004) $821-852$.

[31] M. Dumbser, A. Hidalgo, M. Castro, C. Parés, E. F. Toro, FORCE schemes on unstructured meshes II: Non-conservative hyperbolic systems, Computer Methods in Applied Mechanics and Engineering 199 (9) (2010) 625-647.

[32] A. Canestrelli, A. Siviglia, M. Dumbser, E. F. Toro, Well-balanced high-order centred schemes for non-conservative hyperbolic systems. Applications to shallow water equations with fixed and mobile bed, Advances in Water Resources 32 (6) (2009) 834-844, ISSN 0309-1708, doi:10.1016/j.advwatres.2009.02.006, URL http://www.sciencedirect.com/science/article/pii/S0309170809000256.

[33] A. Canestrelli, M. Dumbser, A. Siviglia, E. F. Toro, Well-balanced high-order centered schemes on unstructured meshes for shallow water equations with fixed and mobile bed, Advances in Water Resources 33 (3) (2010) 291-303.

[34] M. Castro, J. Gallardo, C. Parés, High order finite volume schemes based on reconstruction of states for solving hyperbolic systems with nonconservative products. Applications to shallowwater systems, Mathematics of computation 75 (255) (2006) 1103-1134.

[35] C. wang Shu, C. wang Shu, Essentially non-oscillatory and weighted essentially non-oscillatory schemes for hyperbolic conservation laws, null 1697 (1998) 325—432, doi:10.1.1.35.6198, URL http://citeseerx.ist.psu.edu/viewdoc/summary?doi=10.1.1.35.6198. 
[36] E. D. Fernández-Nieto, Modelling and numerical simulation of submarine sediment shallow flows: transport and avalanches, Bol. Soc. Esp. Mat. Apl. SëMA 49 (2009) 83-103, ISSN 1575-9822.

[37] C. Berthon, S. Cordier, O. Delestre, M.-H. Le, An analytical solution of the Shallow water system coupled to the Exner equation, Comptes Rendus Mathématique 350 (3-4) (2012) 183-186.

[38] B. Andreotti, P. Claudin, S. Douady, Selection of dune shapes and velocities. Part 2: A two-dimensional modelling, The European Physical Journal B 28 (2002) 341-352, doi: 10.1140/epjb/e2002-00237-3.

[39] M. Bellal, B. Spinewine, C. Savary, Y. Zech, Morphological evolution of steep-sloped river beds in the presence of a hydraulic jump: Experimental study, in: XXX IAHR Congress, 133-140, 2003. 